\title{
Existence of a critical point for the infinite divisibility of squares of Gaussian vectors in $R^{2}$ with non-zero mean
}

\author{
Michael B. Marcus Jay Rosen *
}

November 19, 2018

\begin{abstract}
Let $G=\left(G_{1}, G_{2}\right)$ be a Gaussian vector in $R^{2}$ with $E G_{1} G_{2} \neq 0$. Let $c_{1}, c_{2} \in R^{1}$. A necessary and sufficient condition for $G=\left(\left(G_{1}+\right.\right.$ $\left.\left.c_{1} \alpha\right)^{2},\left(G_{2}+c_{2} \alpha\right)^{2}\right)$ to be infinitely divisible for all $\alpha \in R^{1}$ is that

$$
\Gamma_{i, i} \geq \frac{c_{i}}{c_{j}} \Gamma_{i, j}>0 \quad \forall 1 \leq i \neq j \leq 2 .
$$

In this paper we show that when (0.1) does not hold there exists an $0<\alpha_{0}<\infty$ such that $G=\left(\left(G_{1}+c_{1} \alpha\right)^{2},\left(G_{2}+c_{2} \alpha\right)^{2}\right)$ is infinitely divisible for all $|\alpha| \leq \alpha_{0}$ but not for any $|\alpha|>\alpha_{0}$.
\end{abstract}

\section{Introduction}

Let $\eta=\left(\eta_{1}, \ldots, \eta_{n}\right)$ be an $R^{n}$ valued Gaussian random variable. $\eta$ is said to have infinitely divisible squares if $\eta^{2}:=\left(\eta_{1}^{2}, \ldots, \eta_{n}^{2}\right)$ is infinitely divisible, i.e. for any $r$ we can find an $R^{n}$ valued random vector $Z_{r}$ such that

$$
\eta^{2} \stackrel{\text { law }}{=} Z_{r, 1}+\cdots+Z_{r, r}
$$

where $\left\{Z_{r, j}\right\}, j=1, \ldots, r$ are independent identically distributed copies of $Z_{r}$. We express this by saying that $\eta^{2}$ is infinitely divisible.

*Research of both authors supported by grants from the National Science Foundation and PSCCUNY. 
Paul Lévy proposed the problem of characterizing which Gaussian vectors have infinitely divisible squares. It is easy to see that a single Gaussian random variable has infinitely divisible squares. However, even for vectors in $R^{2}$ this is a difficult problem. It seems that Lévy incorrectly conjectured that not all Gaussian vectors in $R^{2}$ have infinitely divisible squares. If he had said $R^{3}$ his conjecture would have been correct.

Lévy's problem was solved by Griffiths and Bapapt [1, 7], (see also [9, Theorem 13.2.1]).

Theorem 1.1 Let $G=\left(G_{1}, \ldots, G_{n}\right)$ be a mean zero Gaussian random variable with strictly positive definite covariance matrix $\Gamma=\left\{\Gamma_{i, j}\right\}=\left\{E\left(G_{i} G_{j}\right)\right\}$. Then $G^{2}$ is infinitely divisible if and only if there exists a signature matrix $\mathcal{N}$ such that

$$
\mathcal{N} \Gamma^{-1} \mathcal{N} \quad \text { is an } M \text { matrix. }
$$

We need to define the different types of matrices that appear in this theorem. Let $A=\left\{a_{i, j}\right\}_{1 \leq i, j \leq n}$ be an $n \times n$ matrix. We call $A$ a positive matrix and write $A \geq 0$ if $a_{i, j} \geq 0$ for all $i, j$. We say that $A$ has positive row sums if $\sum_{j=1}^{n} a_{i, j} \geq 0$ for all $1 \leq i \leq n$.

The matrix $A$ is said to be an $M$ matrix if

(1) $a_{i, j} \leq 0$ for all $i \neq j$.

(2) $A$ is nonsingular and $A^{-1} \geq 0$.

A matrix is called a signature matrix if its off-diagonal entries are all zero and its diagonal entries are either one or minus one. The role of the signature matrix is easy to understand. It simply accounts for the fact that if $G$ has an infinitely divisible square, then so does $\left(\epsilon_{1} G_{1}, \ldots, \epsilon_{n} G_{n}\right)$ for any choice of $\epsilon_{i}= \pm 1, i=1, \ldots, n$. Therefore, if (1.2) holds for $\mathcal{N}$ with diagonal elements $n_{1}, \ldots, n_{n}$

$$
\left(\mathcal{N} \Gamma^{-1} \mathcal{N}\right)^{-1}=\mathcal{N} \Gamma \mathcal{N} \geq 0
$$

since the inverse of an $M$ matrix is positive. Thus $\left(n_{1} G_{1}, \ldots, n_{n} G_{n}\right)$ has a positive covariance matrix and its inverse is an $M$ matrix. (For this reason, in studying mean zero Gaussian vectors with infinitely divisible squares one can restrict ones attention to vectors with positive covariance.)

The natural next step was to characterize Gaussian processes with infinitely divisible squares which do not have mean zero. We set $\eta_{i}=G_{i}+c_{i}$, 
$E G_{i}=0, i=1, \ldots, n$. Let $\Gamma$ be the covariance matrix of $\left(G_{1}, \ldots, G_{n}\right)$ and set

$$
c:=\left(c_{1}, \ldots, c_{n}\right)
$$

Set

$$
G+c:=\left(G_{1}+c_{1}, \ldots, G_{n}+c_{n}\right)
$$

and

$$
(G+c)^{2}:=\left(\left(G_{1}+c_{1}\right)^{2}, \ldots,\left(G_{n}+c_{n}\right)^{2}\right) .
$$

Results about the infinite divisibility of $(G+c)^{2}$ when $c_{1}=\cdots=c_{n}$, are given in the work of N. Eisenbaum [2, 3] and then in joint work by Eisenbaum and H. Kaspi [4], as a by product of their characterization of Gaussian processes with a covariance that is the 0-potential density of a symmetric Markov process. We point out later in this Introduction how Gaussian vectors with infinitely divisible squares are related to the local times of the Markov chain that is determined by the covariance of the Gaussian vector. It is this connection between Gaussian vectors with infinitely divisible squares and the local times of Markov chains, and more generally, between Gaussian processes with infinitely divisible squares and the local times of Markov processes, that enhances our interest in the question of characterizing Gaussian vectors with infinitely divisible squares.

Some of the results in [2, 3, 4] are presented and expanded in [9, Chapter 13]. The following theorem is taken from [9, Theorem 13.3.1 and Lemma $13.3 .2]$.

Theorem 1.2 Let $G=\left(G_{1}, \ldots, G_{n}\right)$ be a mean zero Gaussian random variable with strictly positive definite covariance matrix $\Gamma=\left\{\Gamma_{i, j}\right\}=\left\{E\left(G_{i} G_{j}\right)\right\}$. Let $\mathbf{1}=(1, \ldots, 1) \in R^{n}$. Then the following are equivalent:

(1) $(G+1 \alpha)$ has infinitely divisible squares for all $\alpha \in R^{1}$;

(2) For $\xi=N\left(0, b^{2}\right)$ independent of $G,\left(G_{1}+\xi, \ldots, G_{n}+\xi\right.$, $\left.\xi\right)$ has infinitely divisible squares for some $b \neq 0$. Furthermore, if this holds for some $b \neq 0$, it holds for all $b \in R^{1}$, with $N(0,0)=0$.

(3) $\Gamma^{-1}$ is an $M$ matrix with positive row sums.

In [8], Theorem 1.2 is generalized so that the mean of the components of $G+c$ in (1.5) need not be the same. In this generalization certain trivial cases spoil the simplicity of the final result. We avoid them by requiring that the covariance matrix of the Gaussian process is irreducible. 
Theorem 1.3 Let $G=\left(G_{1}, \ldots, G_{n}\right)$ be a mean zero Gaussian random variable with irreducible strictly positive definite covariance matrix $\Gamma=\left\{\Gamma_{i, j}\right\}=$ $\left\{E\left(G_{i} G_{j}\right)\right\}$. Let $c=\left(c_{1}, \ldots, c_{n}\right) \in R^{n}, c \neq \mathbf{0}$ and let $C$ be a diagonal matrix with $c_{i}=C_{i, i}, 1 \leq i \leq n$. Then the following are equivalent:

(1) $G+c \alpha$ has infinitely divisible squares for all $\alpha \in R^{1}$;

(2) For $\xi=N\left(0, b^{2}\right)$ independent of $G,\left(G_{1}+c_{1} \xi, \ldots, G_{n}+c_{n} \xi\right.$, $\left.\xi\right)$ has infinitely divisible squares for some $b \neq 0$. Furthermore, if this holds for some $b \neq 0$, it holds for all $b \in R^{1}$;

(3) $C \Gamma^{-1} C$ is an $M$ matrix with positive row sums.

By definition, when $(G+c)^{2}$ is infinitely divisible, it can be written as in (1.1) as a sum of $r$ independent identically distributed random variables, for all $r \geq 1$. Based on the work of Eisenbaum and Kaspi mentioned above and the joint paper [5] we can actually describe the decomposition. We give a rough description here. For details see [2, 3, 4] and [9, Chapter 13].

Assume that (1), (2) and (3) of Theorem 1.3 hold. Let

$$
\frac{G}{c}=\left(\frac{G_{1}}{c_{1}}, \ldots, \frac{G_{n}}{c_{n}}\right)
$$

Let $\Gamma_{c}$ denote the covariance matrix of $G / c$. Theorem 1.2 holds for $G / c$ and $\Gamma_{c}$, so $\Gamma_{c}^{-1}$ is an $M$ matrix with positive row sums. To be specific let $G / c \in R^{n}$. Set $S=\{1, \ldots, n\}$. By [9, Theorem 13.1.2], $\Gamma_{c}$ is the 0-potential density of a strongly symmetric transient Borel right process, say $X$, on $S$. We show in the proof of [9, Theorem 13.3.1] that we can find a strongly symmetric recurrent Borel right process $Y$ on $S \cup\{0\}$ with $P^{x}\left(T_{0}<\infty\right)>0$ for all $x \in S$ such that $X$ is the process obtained by killing $Y$ the first time it hits 0 . Let $L_{t}^{x}=\left\{L_{t}^{x} ; t \in R_{+}, x \in S \cup\{0\}\right\}$ denote the local time of $Y$. It follows from the generalized second Ray-Knight Theorem in [5], see also [9, Theorem 8.2.2] that under $P^{0} \times P_{G}$,

$$
\left\{L_{\tau(t)}^{x}+\frac{1}{2}\left(\frac{G_{x}}{c_{x}}\right)^{2} ; x \in S\right\} \stackrel{\operatorname{law}}{=}\left\{\frac{1}{2}\left(\frac{G_{x}}{c_{x}}+\sqrt{2 t}\right)^{2} ; x \in S\right\}
$$

for all $t \in R_{+}$, where $\tau(t)=\inf \left\{s>0 \mid L_{s}^{0}>t\right\}$, the inverse local time at zero, and $Y$ and $G$ are independent. Consequently

$$
\left\{c_{x}^{2} L_{\tau\left(\alpha^{2} / 2\right)}^{x}+\frac{1}{2} G_{x}^{2} ; x \in S\right\} \stackrel{\text { law }}{=}\left\{\frac{1}{2}\left(G_{x}+c_{x} \alpha\right)^{2} ; x \in S\right\}
$$


for all $\alpha \in R^{1}$. (We can extend $\alpha$ from $R_{+}$to $R^{1}$ because $G$ is symmetric.) $\left\{c_{x}^{2} L_{\tau\left(\alpha^{2} / 2\right)}^{x} ; x \in S\right\}$ and $\left\{\frac{1}{2} G_{x}^{2} ; x \in S\right\}$ are independent. $G^{2}$ is infinitely divisible and for all integers $r \geq 1$

$$
c^{2} L_{\tau\left(\alpha^{2} / 2\right)} \stackrel{\text { law }}{=} c^{2} L_{\tau\left(\alpha^{2} /(2 r)\right), 1}^{\cdot}+\cdots+c^{2} L_{\tau\left(\alpha^{2} /(2 r)\right), r}^{\cdot}
$$

where $\left\{L_{\tau\left(\alpha^{2} /(2 r)\right), j}^{\cdot}\right\}, j=1, \ldots, r$ are independent.

Note that in (1.9) we identify the components of the decomposition of $\left\{\left(G_{x}+c_{x} \alpha\right)^{2} ; x \in S\right\}$ that mark it as infinitely divisible.

In Theorem 1.3 we have necessary and sufficient conditions for $\left(\left(G_{1}+\right.\right.$ $\left.\left.c_{1} \alpha\right)^{2},\left(G_{2}+c_{2} \alpha\right)^{2}\right)$ to be infinitely divisible for all $\alpha \in R^{1}$. There remains the question, can $\left(\left(G_{1}+c_{1} \alpha\right)^{2},\left(G_{2}+c_{2} \alpha\right)^{2}\right)$ have infinitely divisible squares for some $\alpha>0$ but not for all $\alpha \in R^{1}$ ? When we began to investigate this question we hoped that such points $\alpha$ do not exist. This would have finished off the problem of characterizing Gaussian random variables with infinitely divisible squares and, more significantly, by (1.9), would show that when a Gaussian random variable with non-zero mean has infinitely divisible squares, it decomposes into the sum of two independent random variables. The Gaussian random variable itself minus its mean, and the local time of a related Markov process. This would be a very neat result indeed, but it is not true.

For all Gaussian random variables $\left(G_{1}, G_{2}\right)$ in $R^{2}$ and all $c_{1}, c_{2} \in R^{1}$ define

$$
\mathcal{G}^{2}\left(c_{1}, c_{2}, \alpha\right):=\left(\left(G_{1}+c_{1} \alpha\right)^{2},\left(G_{2}+c_{2} \alpha\right)^{2}\right) .
$$

It follows from Theorem [1.3, (for details see [8, Corollary 1.3, 4.]), that $\mathcal{G}^{2}\left(c_{1}, c_{2}, \alpha\right)$ has infinitely divisible squares for all $\alpha \in R^{1}$ if and only if

$$
\Gamma_{1,1} \geq \frac{c_{1}}{c_{2}} \Gamma_{1,2} \quad \text { and } \quad \Gamma_{2,2} \geq \frac{c_{2}}{c_{1}} \Gamma_{1,2} .
$$

If (1.12) does not hold, we call $0<\alpha_{0}<\infty$ a critical point for the infinite divisibility of $\mathcal{G}^{2}\left(c_{1}, c_{2}, \alpha\right)$ if $\mathcal{G}^{2}\left(c_{1}, c_{2}, \alpha\right)$ is infinitely divisible for all $|\alpha| \leq \alpha_{0}$, and is not infinitely divisible for any $|\alpha|>\alpha_{0}$. In this paper we prove the following theorem:

Theorem 1.4 For all Gaussian random variables $\left(G_{1}, G_{2}\right)$ in $R^{2}$ and all $\left(c_{1}, c_{2}\right) \in R^{2}$ for which (1.12) does not hold, $\mathcal{G}^{2}\left(c_{1}, c_{2}, \alpha\right)$ has a critical point. 
Note that in Theorem 1.4 we consider all $\left(c_{1}, c_{2}\right) \in R^{2}$. It follows from (1.12) that when $E G_{1} G_{2}>0$, then $\mathcal{G}^{2}\left(c_{1}, c_{2}, \alpha\right)$ has infinitely divisible squares for all $\alpha \in R^{1}$ only if $c_{1} c_{2}>0$. Nevertheless, by Theorem 1.4, even when $c_{1} c_{2} \leq 0,\left(G_{1}+c_{1} \alpha, G_{2}+c_{2} \alpha\right)$ does have infinitely divisible squares for $|\alpha|$ sufficiently small.

To conclude this Introduction we explain how we approach the problem of showing that $(G+c \alpha)^{2}$ is infinitely divisible for all $\alpha \in R^{1}$ or only for some $\alpha \in R^{1}$. Since we can only prove Theorem 1.4 for Gaussian random variables in $R^{2}$ we stick to this case, although a similar analysis applies to $R^{n}$ valued Gaussian random variables.

Let $\Gamma$ be the covariance matrix of $G$ and

$$
\widetilde{\Gamma}:=(I+\Gamma \Lambda)^{-1} \Gamma=\left(\Gamma^{-1}+\Lambda\right)^{-1},
$$

where

$$
\Lambda=\left(\begin{array}{cc}
\lambda_{1} & 0 \\
0 & \lambda_{2}
\end{array}\right)
$$

Consider the Laplace transform of $\left(\left(G_{1}+c_{1} \alpha\right)^{2},\left(G_{2}+c_{2} \alpha\right)^{2}\right)$,

$$
\begin{aligned}
E_{G} & \left(e^{-\left(\lambda_{1}\left(G_{1}+c_{1} \alpha\right)^{2}+\lambda_{2}\left(G_{2}+c_{2} \alpha\right)^{2}\right) / 2}\right) \\
& =\frac{1}{(\operatorname{det}(I+\Gamma \Lambda))^{1 / 2}} \exp \left(-\frac{\alpha^{2}}{2}\left(c_{1}^{2} \lambda_{1}+c_{2}^{2} \lambda_{2}-\sum_{i, j=1}^{2} c_{i} c_{j} \lambda_{i} \lambda_{j} \widetilde{\Gamma}_{i, j}\right)\right) .
\end{aligned}
$$

(See [9, Lemma 5.2.1].) Set $\lambda_{1}=t\left(1-s_{1}\right)$ and $\lambda_{2}=t\left(1-s_{2}\right), 0 \leq s_{1}, s_{2} \leq 1$ and write (1.15) as

$$
\exp \left(U\left(s_{1}, s_{2}, t, \Gamma\right)+\alpha^{2} V\left(s_{1}, s_{2}, t, c_{1}, c_{2}, \widetilde{\Gamma}\right)\right)
$$

where $U:=U\left(s_{1}, s_{2}, t, \Gamma\right)=-1 / 2 \log (\operatorname{det}(I+\Gamma \Lambda))$. Note that $\exp \left(U\left(s_{1}, s_{2}, t, \Gamma\right)\right)$ is the Laplace transform of $\left(G_{1}^{2}, G_{2}^{2}\right)$, with the change of variables $\lambda_{1}=t\left(1-s_{1}\right)$ and $\lambda_{2}=t\left(1-s_{2}\right)$. It is easy to see from Theorem 1.1 that all two dimensional Gaussian random variables are infinitely divisible. Therefore, for all $t$ sufficiently large, all the coefficients of the power series expansion of $U$ in $s_{1}$ and $s_{2}$ are positive, except for the constant term. This is a necessary and sufficient condition for a function to be the Laplace transform of an infinitely divisible random variable. See e.g. [9, Lemma 13.2.2].

Now, suppose that for all $t$ sufficiently large, $V\left(s_{1}, s_{2}, t, c_{1}, c_{2}, \widetilde{\Gamma}\right)$ has all the coefficients of its power series expansion in $s_{1}$ and $s_{2}$ positive, except 
for the constant term. Then the right-hand side of (1.15) is the Laplace transform of two independent infinitely divisible random variables. It is completely obvious that this holds for all $\alpha \in R^{1}$.

On the other hand suppose that for all $t$ sufficiently large, the power series expansion of $V\left(s_{1}, s_{2}, t, c_{1}, c_{2}, \widetilde{\Gamma}\right)$ in $s_{1}$ and $s_{2}$ has even one negative coefficient, besides the coefficient of the constant term. Then for all $\alpha$ sufficiently large, (1.16) is not the Laplace transform of an infinitely divisible random variable. In other words $\left(\left(G_{1}+c_{1} \alpha\right)^{2},\left(G_{2}+c_{2} \alpha\right)^{2}\right)$ is not infinitely divisible for all $\alpha \in R^{1}$. But it may be infinitely divisible if $\alpha$ is small, since the positive coefficients of $\mathrm{U}$ may be greater than or equal to $\alpha^{2}$ times the corresponding negative coefficients of $V$. Clearly, if this is true for some $|\alpha|=\alpha_{0}>0$, then it is true for all $|\alpha| \leq \alpha_{0}$.

The preceding paragraph explains how we prove Theorem 1.4. We consider vectors $\left(\left(G_{1}+c_{1} \alpha\right)^{2},\left(G_{2}+c_{2} \alpha\right)^{2}\right)$ that are not infinitely divisible for all $\alpha \in R^{1}$, (this is easy to do using (1.12)), and show that for $|\alpha|$ sufficiently small the coefficients in the power series expansion of

$$
U\left(s_{1}, s_{2}, t, \Gamma\right)+\alpha^{2} V\left(s_{1}, s_{2}, t, c_{1}, c_{2}, \widetilde{\Gamma}\right)
$$

in $s_{1}$ and $s_{2}$ are positive, except for the constant term. Our proof only uses elementary mathematics, although it is quite long and complicated. In the course of the proof we show that the coefficients of the power series expansion of $U$ in $s_{1}$ and $s_{2}$ are positive, except for the constant term. This provides a direct elementary proof of the fact that the Gaussian random variable $\left(G_{1}, G_{2}\right)$ always has infinitely divisible squares.

As we have just stated, and as the reader will see, the proof of Theorem 1.4 is long and complicated. So far we have not been able to extend it to apply to Gaussian random variables in $R^{3}$. One hopes for a more sophisticated and much shorter proof of Theorem 1.4 that doesn't depend on the dimension of the Gaussian random variable.

\section{Gaussian squares in $R^{2}$ and their Laplace transforms}

Let $G=\left(G_{1}, G_{2}\right)$ be a mean zero Gaussian process with covariance matrix

$$
\Gamma=\left(\begin{array}{ll}
a & 1 \\
1 & b
\end{array}\right)
$$


where $a b=d+1>1$, and let $G+c:=\left(G_{1}+c_{1}, G_{2}+c_{2}\right), c_{1}, c_{2} \in R^{1}$. Note that

$$
\operatorname{det} \Gamma=d
$$

and

$$
\Gamma^{-1}=\frac{1}{d}\left(\begin{array}{cc}
b & -1 \\
-1 & a
\end{array}\right)
$$

Let

$$
\Lambda=\left(\begin{array}{cc}
\lambda_{1} & 0 \\
0 & \lambda_{2}
\end{array}\right)
$$

Then

$$
\Gamma^{-1}+\Lambda=\frac{1}{d}\left(\begin{array}{cc}
b+d \lambda_{1} & -1 \\
-1 & a+d \lambda_{2}
\end{array}\right)
$$

and

$$
\begin{aligned}
\widetilde{\Gamma} & :=(I+\Gamma \Lambda)^{-1} \Gamma=\left(\Gamma^{-1}+\Lambda\right)^{-1} \\
& =d\left(\begin{array}{cc}
b+d \lambda_{1} & -1 \\
-1 & a+d \lambda_{2}
\end{array}\right)^{-1} \\
& =\frac{1}{H\left(a, b, \lambda_{1}, \lambda_{2}\right)}\left(\begin{array}{cc}
\lambda_{2} d+a & 1 \\
1 & \lambda_{1} d+b
\end{array}\right)
\end{aligned}
$$

where

$$
H\left(a, b, \lambda_{1}, \lambda_{2}\right)=1+a \lambda_{1}+b \lambda_{2}+d \lambda_{1} \lambda_{2}=d \operatorname{det}\left(\Gamma^{-1}+\Lambda\right) .
$$

(We use repeatedly the fact that $a b=d+1$.) Note that by $(2.2)$ we have that

$$
\operatorname{det}(I+\Gamma \Lambda)=\operatorname{det}\left(\Gamma\left(\Gamma^{-1}+\Lambda\right)\right)=d \operatorname{det}\left(\Gamma^{-1}+\Lambda\right)=H\left(a, b, \lambda_{1}, \lambda_{2}\right) .
$$

\section{Lemma 2.1}

$$
\begin{aligned}
E_{G} & \left(e^{-\left(\lambda_{1}\left(G_{1}+c_{1}\right)^{2}+\lambda_{2}\left(G_{2}+c_{2}\right)^{2}\right) / 2}\right) \\
& =\frac{1}{\left(H\left(a, b, \lambda_{1}, \lambda_{2}\right)\right)^{1 / 2}} \exp \left(-\frac{c_{1}^{2} \lambda_{1}+c_{2}^{2} \lambda_{2}+\left(c_{1}^{2} b+c_{2}^{2} a-2 c_{1} c_{2}\right) \lambda_{1} \lambda_{2}}{2 H\left(a, b, \lambda_{1}, \lambda_{2}\right)}\right) .
\end{aligned}
$$


Proof By [9, Lemma 5.2.1]

$$
\begin{aligned}
& E_{G}\left(e^{-\left(\lambda_{1}\left(G_{1}+c_{1}\right)^{2}+\lambda_{2}\left(G_{2}+c_{2}\right)^{2}\right) / 2}\right) \\
& =\frac{1}{\left(H\left(a, b, \lambda_{1}, \lambda_{2}\right)\right)^{1 / 2}} \exp \left(-\frac{1}{2}\left(c_{1}^{2} \lambda_{1}+c_{2}^{2} \lambda_{2}\right.\right. \\
& \left.\left.\quad-\frac{c_{1}^{2} \lambda_{1}^{2}\left(\lambda_{2} d+a\right)+2 c_{1} c_{2} \lambda_{1} \lambda_{2}+c_{2}^{2} \lambda_{2}^{2}\left(\lambda_{1} d+b\right)}{H\left(a, b, \lambda_{1}, \lambda_{2}\right)}\right)\right) .
\end{aligned}
$$

A simple computation shows that

$$
\begin{aligned}
\left(c_{1}^{2} \lambda_{1}+c_{2}^{2} \lambda_{2}\right) & H\left(a, b, \lambda_{1}, \lambda_{2}\right) \\
& -\left(c_{1}^{2} \lambda_{1}^{2}\left(\lambda_{2} d+a\right)+2 c_{1} c_{2} \lambda_{1} \lambda_{2}+c_{2}^{2} \lambda_{2}^{2}\left(\lambda_{1} d+b\right)\right) \\
=c_{1}^{2} \lambda_{1}+ & c_{2}^{2} \lambda_{2}+\left(c_{1}^{2} b+c_{2}^{2} a-2 c_{1} c_{2}\right) \lambda_{1} \lambda_{2},
\end{aligned}
$$

from which we get (2.9) .

The term $1 /\left(H\left(a, b, \lambda_{1}, \lambda_{2}\right)\right)^{1 / 2}$ is the Laplace transform of $\left(G_{1}^{2}, G_{2}^{2}\right) / 2$ and by [8, Corollary 1.1, 2.] it is the Laplace transform of an infinitely divisible random variable. The exponential term may or may not be a Laplace transform. In fact, by (1.12), we know it is the Laplace transform of an infinitely divisible random variable, for all $\left\{c_{1} \alpha, c_{2} \alpha\right\}$, for all $\alpha \in R^{1}$, if and only if

$$
a \geq \frac{c_{1}}{c_{2}}>0 \quad \text { and } \quad b \geq \frac{c_{2}}{c_{1}}>0 .
$$

To prove Theorem 1.4 we must show that when (2.12) does not hold, there exists an $0<\alpha_{0}<\infty$ such that (2.9) is the Laplace transform of an infinitely divisible random variable when $c_{1}$ and $c_{2}$ are replaced by $c_{1} \alpha$ and $c_{2} \alpha$ for any $|\alpha| \leq \alpha_{0}$. Actually, as we see in Section 8 , the general result follows from the consideration of three cases,

1. $c_{1}=c_{2}:=(c, c)$;

2. $c_{1}=-c_{2}:=(c,-c)$

3. $(c, 0)$.

This is because if $c_{1} \neq c_{2}$ and neither of them is zero, we can replace $\left(G_{1}, G_{2}\right)$ by $\left(G_{1} /\left|c_{1}\right|, G_{2} /\left|c_{2}\right|\right)$. Clearly, in this case, if Theorem 1.4 holds for $\left(G_{1} /\left|c_{1}\right|, G_{2} /\left|c_{2}\right|\right)$ it holds for $\left(G_{1}, G_{2}\right)$.

In these three cases the numerator of the fraction in the exponential term on the right-hand side of (2.9) is 
1. $c^{2}\left((a+b-2) \lambda_{1} \lambda_{2}+\lambda_{1}+\lambda_{2}\right)$;

2. $c^{2}\left((a+b+2) \lambda_{1} \lambda_{2}+\lambda_{1}+\lambda_{2}\right)$

3. $c^{2}\left(b \lambda_{1} \lambda_{2}+\lambda_{1}\right)$.

Set

$$
\gamma=a+b-2 \quad \text { and } \quad \rho=a+b+2 .
$$

Note unless $\operatorname{det} \Gamma=0, a b>1$. Since Theorem 1.4 obviously holds when $\operatorname{det} \Gamma=0$, we can exclude this case from further consideration. Thus we always have $\gamma>0$.

\section{Power series expansion of the logarithm of the Laplace transform of $\left(\left(\mathbf{G}_{1}+\mathbf{c}\right)^{2},\left(\mathbf{G}_{2}+\mathbf{c}\right)^{2}\right)$ when $\mathrm{EG}_{1} \mathrm{G}_{2}=1$}

Bapat's proof of Theorem 1.1 involves the analysis of a certain power series expansion of the logarithm of the Laplace transform. We need a similar, but more delicate, analysis. (See Lemma 4.1 below).

Using (2.9) and the remarks following Lemma 2.1 we can write

$$
\begin{aligned}
E_{G}\left(e^{-\left(\lambda_{1}\left(G_{1}+c\right)^{2}+\lambda_{2}\left(G_{2}+c\right)^{2}\right) / 2}\right) & \\
& =\exp \left(-\frac{1}{2} \log H\left(a, b, \lambda_{1}, \lambda_{2}\right)\right) \exp \left(-\frac{c^{2}\left(\gamma \lambda_{1} \lambda_{2}+\lambda_{1}+\lambda_{2}\right)}{2 H\left(a, b, \lambda_{1}, \lambda_{2}\right)}\right) . \\
& :=\exp \left(\frac{1}{2}\left(P\left(a, b, \lambda_{1}, \lambda_{2}\right)+c^{2} Q\left(a, b, \lambda_{1}, \lambda_{2}\right)\right)\right),
\end{aligned}
$$

Since $a b=d+1$, (recall that $d>0$ ), we have

$$
\begin{aligned}
a+b-(d+2) & =a+\frac{(d+1)}{a}-(d+2) \\
& =\frac{1}{a}\left(a^{2}-(d+2) a+(d+1)\right) \\
& =\frac{1}{a}(a-(d+1))(a-1) .
\end{aligned}
$$

Thus $a+b-(d+2) \leq 0$ if and only if $1 \leq a \leq d+1$, which in view of $a b=d+1$ is equivalent to $1 \leq b \leq d+1$. Consequently (2.12) holds if and only if $a+b-(d+2) \leq 0$. 
Therefore, to show that $\left(\left(G_{1}+c\right)^{2},\left(G_{2}+c\right)^{2}\right)$ is infinitely divisible, for some, but not for all, $c>0$, we must consider $a, b>0$ such that

$$
\zeta:=a+b-(d+2)>0 .
$$

In the rest of this paper we assume that (3.3) holds.

Let $\lambda_{1}=t\left(1-s_{1}\right)$ and $\lambda_{2}=t\left(1-s_{2}\right), 0 \leq s_{1}, s_{2} \leq 1$. We consider $P$ and $Q$ as functions of and $s_{1}, s_{2}, t$, and write

$$
P\left(s_{1}, s_{2}, t\right):=P\left(a, b, \lambda_{1}, \lambda_{2}\right), \quad Q\left(s_{1}, s_{2}, t\right):=Q\left(a, b, \lambda_{1}, \lambda_{2}\right) .
$$

We expand these in a power series in $s_{1}, s_{2}$.

$$
P\left(s_{1}, s_{2}, t\right)=\sum_{j, k=0}^{\infty} P_{j, k}(t) s_{1}^{j} s_{2}^{k}, \quad Q\left(s_{1}, s_{2}, t\right)=\sum_{j, k=0}^{\infty} Q_{j, k}(t) s_{1}^{j} s_{2}^{k},
$$

and set

$$
R\left(s_{1}, s_{2}, t, c\right)=P\left(s_{1}, s_{2}, t\right)+c^{2} Q\left(s_{1}, s_{2}, t, c\right) .
$$

Consequently

$$
R\left(s_{1}, s_{2}, t, c\right)=\sum_{j, k=0}^{\infty} R_{j, k}(t, c) s_{1}^{j} s_{2}^{k}
$$

with

$$
R_{j, k}(t, c)=P_{j, k}(t)+c^{2} Q_{j, k}(t) .
$$

In this section we obtain explicit expressions for $P_{j, k}(t), Q_{j, k}(t)$. We write

$$
\begin{aligned}
H\left(a, b, \lambda_{1}, \lambda_{2}\right) & =1+a \lambda_{1}+b \lambda_{2}+d \lambda_{1} \lambda_{2} \\
& =1+a t+b t+d t^{2}-\left(a t+d t^{2}\right) s_{1}-\left(b t+d t^{2}\right) s_{2}+d t^{2} s_{1} s_{2} \\
& =\left(1+a t+b t+d t^{2}\right)\left(1-\alpha s_{1}-\beta s_{2}+p s_{1} s_{2}\right) \\
& =\left(1+a t+b t+d t^{2}\right)\left(1-\alpha s_{1}-\beta s_{2}+\theta \alpha \beta s_{1} s_{2}\right)
\end{aligned}
$$

where

$$
\alpha=\frac{a t+d t^{2}}{1+a t+b t+d t^{2}}, \quad \beta=\frac{b t+d t^{2}}{1+a t+b t+d t^{2}}, \quad p=\frac{d t^{2}}{1+a t+b t+d t^{2}}
$$

and

$$
\alpha \beta=p\left(\frac{1+d^{-1}+a t+b t+d t^{2}}{1+a t+b t+d t^{2}}\right):=\frac{p}{\theta} .
$$


Note that

$$
1-\theta=\frac{d^{-1}}{1+d^{-1}+a t+b t+d t^{2}} \leq \frac{1}{d^{2} t^{2}} .
$$

Using these definitions we have

$$
\begin{aligned}
P\left(a, b, \lambda_{1}, \lambda_{2}\right) & =-\log H\left(a, b, \lambda_{1}, \lambda_{2}\right) \\
& =-\log \left(1+a t+b t+d t^{2}\right)-\log \left(1-\alpha s_{1}-\beta s_{2}+\theta \alpha \beta s_{1} s_{2}\right)
\end{aligned}
$$

and

$$
\begin{aligned}
Q\left(a, b, \lambda_{1}, \lambda_{2}\right) & \\
= & -\frac{\gamma \lambda_{1} \lambda_{2}+\lambda_{1}+\lambda_{2}}{H\left(a, b, \lambda_{1}, \lambda_{2}\right)} \\
& =-\frac{1}{\left(1+a t+b t+d t^{2}\right)} \frac{\gamma t^{2}\left(1-s_{1}\right)\left(1-s_{2}\right)+t\left(2-s_{1}-s_{2}\right)}{1-\alpha s_{1}-\beta s_{2}+\theta \alpha \beta s_{1} s_{2}} .
\end{aligned}
$$

We make some preliminary observations that enable us to compute the coefficients of the power series expansions of $P\left(s_{1}, s_{2}, t\right)$ and $Q\left(s_{1}, s_{2}, t\right)$. Let $\left(u_{1}, u_{2}\right) \in[0,1)^{2}$, and $\theta \in[0,1)$, and assume that

$$
u_{1}+u_{2}-\theta u_{1} u_{2}<1
$$

(It is clearly greater than zero.) Let

$$
\frac{1}{1-u_{1}-u_{2}+\theta u_{1} u_{2}}:=\sum_{j, k=0}^{\infty} D_{j, k} u_{1}^{j} u_{2}^{k}
$$

and

$$
-\log \left(1-u_{1}-u_{2}+\theta u_{1} u_{2}\right):=\sum_{j, k=0}^{\infty} C_{j, k} u_{1}^{j} u_{2}^{k} .
$$

We give explicit expressions for $C_{j, k}$ and $D_{j, k}$. To begin we give several equalities that are easy to verify.

Lemma 3.1 For $u \in[0,1)$

$$
\frac{1}{(1-u)^{q}}=\sum_{n=0}^{\infty}\left(\begin{array}{c}
q+n-1 \\
n
\end{array}\right) u^{n} .
$$


Proof To get $\left(\begin{array}{c}q+n-1 \\ n\end{array}\right)$ differentiate $1 /(1-u)^{q}, n$ times, divide by $n$ ! and set $u=0$.

We list the following equalities without proof.

\section{Lemma 3.2}

$$
\begin{aligned}
\left(\begin{array}{l}
k \\
p
\end{array}\right)-\left(\begin{array}{c}
k-1 \\
p
\end{array}\right) & =\left(\begin{array}{l}
k-1 \\
p-1
\end{array}\right) \\
\left(\begin{array}{c}
k-1 \\
p
\end{array}\right) & =\frac{k-p}{k}\left(\begin{array}{l}
k \\
p
\end{array}\right) \\
\left(\begin{array}{l}
k-1 \\
p-1
\end{array}\right) & =\frac{p}{k}\left(\begin{array}{l}
k \\
p
\end{array}\right) \\
\left(\begin{array}{l}
k \\
p
\end{array}\right) & =\frac{k-p+1}{p}\left(\begin{array}{c}
k \\
p-1
\end{array}\right) .
\end{aligned}
$$

Lemma 3.3 For $0 \leq j \leq k$

$$
D_{j, k}=\sum_{p=0}^{j}(1-\theta)^{p}\left(\begin{array}{l}
j \\
p
\end{array}\right)\left(\begin{array}{l}
k \\
p
\end{array}\right) .
$$

Also, $C_{0,0}=0, C_{j, 0}=1 / j, C_{0, k}=1 / k, j, k \neq 0$, and for $1 \leq j \leq k$

$$
C_{j, k}=\sum_{p=0}^{j-1}(1-\theta)^{p}\left(\begin{array}{l}
j \\
p
\end{array}\right)\left(\begin{array}{l}
k \\
p
\end{array}\right) \frac{(1-\theta)}{p+1} \frac{(j-p)(k-p)}{j k} .
$$

Proof Note that

$$
\frac{1}{1-u_{1}-u_{2}+\theta u_{1} u_{2}}=\sum_{n=0}^{\infty}\left(u_{1}+u_{2}-\theta u_{1} u_{2}\right)^{n} .
$$

Writing (3.15) in the form $u_{1}+u_{2}-u_{1} u_{2}+(1-\theta) u_{1} u_{2}<1$ we see that it is equivalent to the statement that

$$
\frac{(1-\theta) u_{1} u_{2}}{\left(1-u_{1}\right)\left(1-u_{2}\right)}<1
$$

We write

$$
\frac{1}{1-u_{1}-u_{2}+\theta u_{1} u_{2}}
$$




$$
\begin{aligned}
& =\left(\left(1-u_{1}\right)\left(1-u_{2}\right)\left(1-\frac{(1-\theta) u_{1} u_{2}}{\left(1-u_{1}\right)\left(1-u_{2}\right)}\right)\right)^{-1} \\
& =\frac{1}{\left(1-u_{1}\right)\left(1-u_{2}\right)} \sum_{p=0}^{\infty}\left(\frac{(1-\theta) u_{1} u_{2}}{\left(1-u_{1}\right)\left(1-u_{2}\right)}\right)^{p} \\
& =\sum_{p=0}^{\infty} \frac{(1-\theta)^{p} u_{1}^{p} u_{2}^{p}}{\left(1-u_{1}\right)^{p+1}\left(1-u_{2}\right)^{p+1}} .
\end{aligned}
$$

Using this series we can determine $D_{j, k}$. Since $j \leq k$ it is clear that each of the first $j+1$ terms in the series immediately above can contribute a term in $u_{1}^{j} u_{2}^{k}$. For a given $0 \leq p \leq j$ we get $(1-\theta)^{p}$ times the coefficient of $u_{1}^{j-p}$ in the power series expansion of $1 /\left(1-u_{1}\right)^{p+1}$ and the coefficient of $u_{2}^{k-p}$ in the power series expansion of $1 /\left(1-u_{2}\right)^{p+1}$. We see from Lemma 3.1 that they are $\left(\begin{array}{c}j \\ j-p\end{array}\right)=\left(\begin{array}{l}j \\ p\end{array}\right)$ and $\left(\begin{array}{c}k \\ k-p\end{array}\right)=\left(\begin{array}{l}k \\ p\end{array}\right)$ respectively. Thus we get (3.20).

To obtain (3.21) we write

$$
\begin{aligned}
& -\log \left(1-u_{1}-u_{2}+\theta u_{1} u_{2}\right) \\
& \quad=-\log \left(\left(1-u_{1}\right)\left(1-u_{2}\right)-(1-\theta) u_{1} u_{2}\right) \\
& \quad=-\log \left(1-u_{1}\right)-\log \left(1-u_{2}\right)-\log \left(1-\frac{(1-\theta) u_{1} u_{2}}{\left(1-u_{1}\right)\left(1-u_{2}\right)}\right) \\
& \quad=\sum_{n=1}^{\infty} \frac{u_{1}^{n}}{n}+\sum_{n=1}^{\infty} \frac{u_{2}^{n}}{n}+\sum_{p=1}^{\infty} \frac{1}{p}\left(\frac{(1-\theta) u_{1} u_{2}}{\left(1-u_{1}\right)\left(1-u_{2}\right)}\right)^{p} .
\end{aligned}
$$

This gives us $C_{j, 0}$ and $C_{0, k}$ and, similar to the computation of $D_{j, k}$, we can use the last series above to see that

$$
\begin{aligned}
C_{j, k} & =\sum_{p=1}^{j} \frac{(1-\theta)^{p}}{p}\left(\begin{array}{c}
j-1 \\
p-1
\end{array}\right)\left(\begin{array}{c}
k-1 \\
p-1
\end{array}\right) \\
& =\sum_{p=0}^{j-1} \frac{(1-\theta)^{p+1}}{p+1}\left(\begin{array}{c}
j-1 \\
p
\end{array}\right)\left(\begin{array}{c}
k-1 \\
p
\end{array}\right) \\
& =\sum_{p=0}^{j-1} \frac{(1-\theta)^{p+1}}{p+1}\left(\begin{array}{c}
j-1 \\
p
\end{array}\right)\left(\begin{array}{c}
k-1 \\
p
\end{array}\right) \\
& =\sum_{p=0}^{j-1}(1-\theta)^{p}\left(\begin{array}{l}
j \\
p
\end{array}\right)\left(\begin{array}{c}
k \\
p
\end{array}\right) \frac{(1-\theta)}{p+1} \frac{(j-p)(k-p)}{j k} .
\end{aligned}
$$

This gives us (3.21). 
Set

$$
\widetilde{t}=\frac{1}{\sqrt{1-\theta}}
$$

By (3.12) we have that

$$
d t \leq \tilde{t} \leq d t+2
$$

for all $t$ sufficiently large.

Lemma 3.4 For all $t$ sufficiently large, $P_{j, 0}(t)=\alpha^{j} / j, P_{0, k}(t)=\beta^{k} / k$ and for all $1 \leq j \leq k$

$$
P_{j, k}(t)=\frac{\alpha^{j} \beta^{k}}{\widetilde{t}^{2}} \sum_{p=0}^{j-1} \widetilde{t}^{-2 p}\left(\begin{array}{l}
j \\
p
\end{array}\right)\left(\begin{array}{l}
k \\
p
\end{array}\right) \frac{1}{p+1} \frac{(j-p)(k-p)}{j k} .
$$

(See (3.5).)

Proof Note that since $0<\alpha, \beta, \theta<1$,

$$
\begin{aligned}
\alpha s_{1}+\beta s_{2}-\theta \alpha \beta s_{1} s_{2} & \leq \alpha+\beta-\theta \alpha \beta \\
& =\alpha+\beta-\alpha \beta+(1-\theta) \alpha \beta \\
& =-(1-\alpha)(1-\beta)+\frac{\alpha \beta}{d^{2} t^{2}}+1+O\left(1 / t^{3}\right) \\
& =-\frac{a b}{d^{2} t^{2}}+\frac{1}{d^{2} t^{2}}+1+O\left(1 / t^{3}\right) \\
& =1-\frac{1}{d^{2} t^{2}}+O\left(1 / t^{3}\right) .
\end{aligned}
$$

Consequently, for all $t$ sufficiently large

$$
0 \leq \alpha s_{1}+\beta s_{2}-\theta \alpha \beta s_{1} s_{2}<1 .
$$

Therefore, by (3.17)

$$
-\log \left(1-\alpha u_{1}-\beta u_{2}+\alpha \beta \theta u_{1} u_{2}\right)=\sum_{j, k=0}^{\infty} \alpha^{j} \beta^{k} C_{j, k} u_{1}^{j} u_{2}^{k} .
$$

The lemma now follows from Lemma 3.3 and (3.27).

Set

$$
\bar{t}=\sqrt{1+a t+b t+d t^{2}}
$$

and note that

$$
d^{1 / 2} t \leq \bar{t} \leq d^{1 / 2} t+2
$$

for all $t$ sufficiently large. 
Lemma 3.5 For all t sufficiently large, and $j, k \geq 1$

$$
Q_{j, 0}(t)=-\frac{\left(\gamma t^{2}(\alpha-1)+t(2 \alpha-1)\right)}{\bar{t}^{2}} \alpha^{j-1}
$$

and

$$
Q_{0, k}(t)=-\frac{\left(\gamma t^{2}(\beta-1)+t(2 \beta-1)\right)}{\bar{t}^{2}} \beta^{j-1} .
$$

Furthermore, for all $t$ sufficiently large and for all $1 \leq j \leq k$

$$
\begin{aligned}
Q_{j, k}(t) & \\
= & \frac{\alpha^{j-1} \beta^{k-1}}{\bar{t}^{2}} \sum_{p=0}^{j} \widetilde{t}^{-2 p}\left(\begin{array}{l}
j \\
p
\end{array}\right)\left(\begin{array}{l}
k \\
p
\end{array}\right) \\
& \left(-\gamma t^{2}\left((1-\alpha)(1-\beta)-\frac{(1-\beta) p}{j}-\frac{(1-\alpha) p}{k}+\widetilde{t}^{-2} \frac{j-p}{j} \frac{k-p}{k}\right)\right. \\
& \left.+t\left(\alpha\left(1-\beta-\frac{p}{k}\right)+\beta\left(1-\alpha-\frac{p}{j}\right)\right)\right) .
\end{aligned}
$$

Proof It follows from (3.31) that for $0 \leq s_{1}, s_{2} \leq 1$

$$
\frac{1}{1+a \lambda_{1}+b \lambda_{2}+d \lambda_{1} \lambda_{2}}=\frac{1}{\bar{t}^{2}} \sum_{n=0}^{\infty}\left(\alpha s_{1}+\beta s_{2}-\theta \alpha \beta s_{1} s_{2}\right)^{n} .
$$

Using this along with (3.14) we see that

$$
\begin{aligned}
Q\left(s_{1}, s_{2}, t\right)= & -\frac{\left(\gamma t^{2}\left(1-s_{1}\right)\left(1-s_{2}\right)+t\left(2-s_{1}-s_{2}\right)\right)}{\bar{t}^{2}} \\
=-\frac{\left(\gamma t^{2}+2 t-\left(\gamma t^{2}+t\right) s_{1}-\left(\gamma t^{2}+t\right) s_{2}+\gamma t^{2} s_{1} s_{2}\right)}{\bar{t}^{2}} & -\sum_{n=0}^{\infty}\left(\alpha s_{1}+\beta s_{2}-\theta \alpha \beta s_{1} s_{2}\right)^{n}
\end{aligned}
$$

Consequently

$$
Q_{j, 0}(t)=-\frac{\left(\left(\gamma t^{2}+2 t\right) \alpha-\left(\gamma t^{2}+t\right)\right)}{\bar{t}^{2}} \alpha^{j-1}
$$


from which we get (3.35), and

$$
Q_{0, k}(t)=-\frac{\left(\left(\gamma t^{2}+2 t\right) \beta-\left(\gamma t^{2}+t\right)\right)}{\bar{t}^{2}} \beta^{j-1},
$$

from which we get (3.36).

To obtain (3.37) we use (3.39), and the terms of $D_{j, k}$ defined in (3.16), to see that

$$
\begin{aligned}
\mathcal{Q}_{j, k}:= & \frac{\bar{t}^{2} Q_{j, k}}{\alpha^{j-1} \beta^{k-1}} \\
= & -\gamma t^{2}\left(D_{j, k} \alpha \beta-D_{j, k-1} \alpha-D_{j-1, k} \beta+D_{j-1, k-1}\right) \\
& \quad-t\left(2 D_{j, k} \alpha \beta-D_{j, k-1} \alpha-D_{j-1, k} \beta\right) .
\end{aligned}
$$

Using (3.42), (3.20) and Lemma 3.2 we see that

$$
\begin{aligned}
\mathcal{Q}_{j, k}=\sum_{p=0}^{j} \tilde{t}^{-2 p}\left(\begin{array}{l}
j \\
p
\end{array}\right)\left(\begin{array}{l}
k \\
p
\end{array}\right) & \left(-\gamma t^{2}\left(\alpha \beta-\alpha \frac{k-p}{k}-\beta \frac{j-p}{j}+\frac{j-p}{j} \frac{k-p}{k}\right)\right. \\
& \left.-t\left(2 \alpha \beta-\alpha \frac{k-p}{k}-\beta \frac{j-p}{j}\right)\right) .
\end{aligned}
$$

Consequently, for all $t$ sufficiently large, for all $1 \leq j \leq k$

$$
\begin{aligned}
Q_{j, k}(t)= & \frac{\alpha^{j-1} \beta^{k-1}}{\bar{t}^{2}} \sum_{p=0}^{j} \widetilde{t}^{-2 p}\left(\begin{array}{l}
j \\
p
\end{array}\right)\left(\begin{array}{l}
k \\
p
\end{array}\right) \\
& \left(-\gamma t^{2}\left(\alpha \beta-\alpha \frac{k-p}{k}-\beta \frac{j-p}{j}+\frac{j-p}{j} \frac{k-p}{k}\right)\right. \\
& \left.-t\left(2 \alpha \beta-\alpha \frac{k-p}{k}-\beta \frac{j-p}{j}\right)\right)
\end{aligned}
$$

Consider (3.44). We write

$$
\begin{aligned}
\alpha \beta- & \alpha \frac{k-p}{k}-\beta \frac{j-p}{j}+\frac{j-p}{j} \frac{k-p}{k} \\
& =(1-\alpha)(1-\beta)-\frac{(1-\beta) p}{j}-\frac{(1-\alpha) p}{k}+\frac{p^{2}}{j k},
\end{aligned}
$$




$$
2 \alpha \beta-\alpha \frac{k-p}{k}-\beta \frac{j-p}{j}=-\alpha\left(1-\beta-\frac{p}{k}\right)-\beta\left(1-\alpha-\frac{p}{j}\right),
$$

to obtain

$$
\begin{aligned}
& Q_{j, k}(t) \\
& =\frac{\alpha^{j-1} \beta^{k-1}}{\bar{t}^{2}} \sum_{p=0}^{j} \tilde{t}^{-2 p}\left(\begin{array}{l}
j \\
p
\end{array}\right)\left(\begin{array}{l}
k \\
p
\end{array}\right) \\
& \left(-\gamma t^{2}\left((1-\alpha)(1-\beta)-\frac{(1-\beta) p}{j}-\frac{(1-\alpha) p}{k}+\frac{p^{2}}{j k}\right)\right. \\
& \left.+t\left(\alpha\left(1-\beta-\frac{p}{k}\right)+\beta\left(1-\alpha-\frac{p}{j}\right)\right)\right) .
\end{aligned}
$$

Note that for $1 \leq q \leq j$

$$
\begin{aligned}
\tilde{t}^{-2 q}\left(\begin{array}{l}
j \\
q
\end{array}\right)\left(\begin{array}{l}
k \\
q
\end{array}\right)\left(\frac{q^{2}}{j k}\right) \\
=\widetilde{t}^{-2(q-1)}\left(\begin{array}{c}
j \\
q-1
\end{array}\right)\left(\begin{array}{c}
k \\
q-1
\end{array}\right) \frac{j-(q-1)}{j} \frac{k-(q-1)}{k} \widetilde{t}^{-2} .
\end{aligned}
$$

Therefore, for each $1 \leq p \leq j$ we incorporate the term in $p^{2} / j k$ in (3.47) into the preceding term in the series in (3.47) to get (3.37). (Note that we can not add anything to the $p=j$ term. The expression in (3.37) reflects this fact since $\frac{j-p}{j} \frac{k-p}{k}=0$ when $p=j$.)

\section{A sufficient condition for a vector in $R^{2}$ to be infinitely divisible.}

We present a sufficient condition for a random vector in $R^{2}$ to be to be infinitely divisible, and show how it simplifies the task of showing that $\left(\left(G_{1}+\right.\right.$ $\left.c)^{2},\left(G_{2}+c\right)^{2}\right)$ is infinitely divisible.

Lemma 4.1 Let $\psi:\left(R_{+}\right)^{2} \rightarrow\left(R_{+}\right)^{2}$ be a continuous function with $\psi(0,0)=$ 1. Let $\mathbf{s} \in[0,1]^{2}$ and suppose that for all $t>0$ sufficiently large, $\log \psi(t(1-$ $\left.\left.s_{1}\right), t\left(1-s_{2}\right)\right)$ has a power series expansion at $\mathbf{s}=\mathbf{0}$ given by

$$
\phi\left(t ; s_{1}, s_{2}\right)=\sum_{j, k=0}^{\infty} b_{j, k}(t) s_{1}^{j} s_{2}^{k}
$$


Suppose also that there exist an increasing sequence of finite subsets $\mathcal{N}_{i} \subseteq$ $\mathbf{N}^{2}, i \geq 1$, with $\bigcup_{i=1}^{\infty} \mathcal{N}_{i}=\mathbf{N}^{2}$, and a sequence $t_{i} \rightarrow \infty, i \geq 1$, such that $b_{j, k}\left(t_{i}\right) \geq 0$ for all $(j, k) \in \mathcal{N}_{i} /(0,0)$ and

$$
\lim _{i \rightarrow \infty} \sum_{j, k \notin N_{i} \cup(0,0)}^{\infty}\left|b_{j, k}\left(t_{i}\right)\right|=0 .
$$

Then $\psi\left(\lambda_{1}, \lambda_{2}\right)$ is the Laplace transform of an infinitely divisible random variable on $\left(R_{+}\right)^{2}$.

Proof It is clear from (4.2) that the power series in (4.1) converges absolutely for all $\mathbf{s} \in[0,1]^{2}$. Let

$$
\phi_{i}\left(t_{i} ; s_{1}, s_{2}\right)=b_{0,0}\left(t_{i}\right)+\sum_{j, k \in N_{i} /(0,0)} b_{j, k}\left(t_{i}\right) s_{1}^{j} s_{2}^{k} .
$$

Set

$$
\Psi_{i}\left(t_{i} ; e^{-\lambda_{1} / t_{i}}, e^{-\lambda_{2} / t_{i}}\right)=\exp \left(\phi_{i}\left(t_{i} ; e^{-\lambda_{1} / t_{i}}, e^{-\lambda_{2} / t_{i}}\right)\right) .
$$

We show that for each $\left(\lambda_{1}, \lambda_{2}\right) \in\left(R_{+}\right)^{2}$

$$
\lim _{i \rightarrow \infty} \Psi_{i}\left(t_{i} ; e^{-\lambda_{1} / t_{i}}, e^{-\lambda_{2} / t_{i}}\right)=\psi\left(\lambda_{1}, \lambda_{2}\right) .
$$

As we point out in [9, page 565], $\Psi_{i}\left(t_{i} ; e^{-\lambda_{1} / t_{i}}, e^{-\lambda_{2} / t_{i}}\right)$ is the Laplace transform of a discrete measure. It then follows from the continuity theorem and the fact that $\psi(0,0)=1$ that $\psi\left(\lambda_{1}, \lambda_{2}\right)$ is the Laplace transform of a random variable. Furthermore repeating this argument with $\phi_{i}\left(t_{i} ; s_{1}, s_{2}\right)$ replaced by $\phi_{i}\left(t_{i} ; s_{1}, s_{2}\right) / n$ shows that $\psi^{1 / n}\left(\lambda_{1}, \lambda_{2}\right)$ is the Laplace transform of a random variable. This shows that $\psi\left(\lambda_{1}, \lambda_{2}\right)$ is the Laplace transform of an infinitely divisible random variable on $\left(R_{+}\right)^{2}$.

Let

$$
\delta_{i}:=\left|\psi\left(t_{i}\left(1-e^{-\lambda_{1} / t_{i}}\right), t_{i}\left(1-e^{-\lambda_{2} / t_{i}}\right)\right)-\psi\left(\lambda_{1}, \lambda_{2}\right)\right| .
$$

Clearly $\lim _{i \rightarrow \infty} \delta_{i}=0$. By (4.2)

$$
\begin{aligned}
& \left|\psi\left(t_{i}\left(1-e^{-\lambda_{1} / t_{i}}\right), t_{i}\left(1-e^{-\lambda_{2} / t_{i}}\right)\right)-\exp \left(\phi_{i}\left(t_{i} ; e^{-\lambda_{1} / t_{i}}, e^{-\lambda_{2} / t_{i}}\right)\right)\right| \\
& =\mid \exp \left(b_{0,0}\left(t_{i}\right)+\sum_{(j, k) \neq(0,0)} b_{j, k}\left(t_{i}\right) e^{-j \lambda_{1} / t_{i}} e^{-k \lambda_{2} / t_{i}}\right)
\end{aligned}
$$




$$
\begin{aligned}
& -\exp \left(b_{0,0}\left(t_{i}\right)+\sum_{j, k \in N_{i} /(0,0)} b_{j, k}\left(t_{i}\right) e^{-j \lambda_{1} / t_{i}} e^{-k \lambda_{2} / t_{i}}\right) \mid \\
= & \psi\left(t_{i}\left(1-e^{-\lambda_{1} / t_{i}}\right), t_{i}\left(1-e^{-\lambda_{2} / t_{i}}\right)\right) \\
& \left|1-\exp \left(-\sum_{j, k \notin \mathcal{N}_{i} \cup(0,0)}^{\infty} b_{j, k}\left(t_{i}\right) e^{-j \lambda_{1} / t_{i}} e^{-k \lambda_{2} / t_{i}}\right)\right| \\
= & \epsilon_{i} \psi\left(t_{i}\left(1-e^{-\lambda_{1} / t_{i}}\right), t_{i}\left(1-e^{-\lambda_{2} / t_{i}}\right)\right),
\end{aligned}
$$

where

$$
\epsilon_{i}:=\left|\left(1-\exp \left(-\sum_{j, k \notin N_{i} \cup(0,0)}^{\infty} b_{j, k}\left(t_{i}\right) e^{-j \lambda_{1} / t_{i}} e^{-k \lambda_{2} / t_{i}}\right)\right)\right| .
$$

Note that by (4.2)

$$
\lim _{i \rightarrow \infty} \epsilon_{i}=0 .
$$

Therefore, by the triangle inequality, (4.6) and (4.9)

$$
\begin{aligned}
& \left|\exp \left(\phi_{i}\left(t_{i} ; e^{-\lambda_{1} / t_{i}}, e^{-\lambda_{2} / t_{i}}\right)\right)-\psi\left(\lambda_{1}, \lambda_{2}\right)\right| \\
& \quad \leq \epsilon_{i} \psi\left(t_{i}\left(1-e^{-\lambda_{1} / t_{i}}\right), t_{i}\left(1-e^{-\lambda_{2} / t_{i}}\right)\right)+\delta_{i} .
\end{aligned}
$$

Using (4.6) we see that this is

$$
\leq \epsilon_{i}\left(\psi\left(\lambda_{1}, \lambda_{2}\right)+\delta_{i}\right)+\delta_{i}
$$

Thus we justify (4.5) and the paragraph following it.

Remark 4.1 In [9, Lemma 13.2.2] we present the well known result that the conclusion of Lemma 4.1 holds when $\log \psi\left(t\left(1-s_{1}\right), t\left(1-s_{2}\right)\right)$ has a power series expansion at $\mathbf{s}=\mathbf{0}$ with all its coefficients, except for the coefficient of the constant term, are positive. Lemma 4.1 is useful because it allows us to only verify this condition for a subset of these coefficients, (depending on $t$ ).

The following lemma enables us to apply Lemma 4.1.

Lemma 4.2 For any $c_{3}>0$ there exists a constant $B=B\left(\gamma, d, c_{3}\right)$ for which

$$
\mathcal{N}_{t}=\{(j, k) \mid \sqrt{j k} \leq B t \log t\}
$$

has the property that

$$
\lim _{t \rightarrow \infty} \sum_{(j, k) \notin \mathcal{N}_{t}}\left|R_{j, k}(t, c)\right|=0
$$

uniformly in $|c| \leq c_{3}$. 
Remark 4.2 It follows from Lemmas 4.1 and 4.2 that in order to prove Theorem 1.4 we need that only show that we can find a $c_{0}>0$, such that

$$
R_{j, k}\left(t, c_{0}\right) \geq 0 \quad \text { for all } \quad \sqrt{j k} \leq B t \log t
$$

for any constant $B$, for all $t$ sufficiently large, (except for $R_{0,0}(t)$ ).

Before proving Lemma 4.2 we establish the following bounds

\section{Lemma 4.3}

$$
\left(\begin{array}{l}
j \\
p
\end{array}\right) \leq\left(\frac{e j}{p}\right)^{p}
$$

and

$$
\frac{1}{\tau^{2 p}}\left(\begin{array}{l}
j \\
p
\end{array}\right)\left(\begin{array}{l}
k \\
p
\end{array}\right) \leq\left(\frac{e \sqrt{j k}}{p \tau}\right)^{2 p} \leq \exp \left(\frac{2 \sqrt{j k}}{\tau}\right)
$$

Proof It is clear that

$$
\left(\begin{array}{l}
j \\
p
\end{array}\right) \leq \frac{j^{p}}{p !}
$$

Therefore to prove (4.3) we need only show that

$$
p !\left(\frac{e}{p}\right)^{p} \geq 1
$$

In [6, page 42 ], Feller shows that $p !(e / p)^{p}$ is increasing in $p$. Since it is equal to $e$ when $p=1$, (and 1 when $p=0$ ), we get (4.17).

The first inequality in (4.15) follows from (4.14) the next one is obtained by maximizing the middle term with respect to $p$.

Proof of Lemma 4.2 By $(3.8)$ and Lemmas 3.4 and 3.5 we see that for all $t$ sufficiently large, for all $1 \leq j \leq k$,

$$
\left|R_{j, k}(t, c)\right| \leq C \alpha^{j} \beta^{k} \sum_{p=0}^{j} \widetilde{t}^{-2 p}\left(\begin{array}{l}
j \\
p
\end{array}\right)\left(\begin{array}{l}
k \\
p
\end{array}\right)
$$

where $C$ depends on $c, \gamma$ and $d$ but not on $j, k, t$ or $\widetilde{t}$. Furthermore, $C$ is bounded for all $|c| \leq T$, for any finite number $T$. (We also use the fact that $\lim _{t \rightarrow \infty} \alpha \beta=1$.) 
For any $\delta>0$, for $t$ sufficiently large,

$$
\alpha=\frac{a t+d t^{2}}{1+a t+b t+d t^{2}}=1-\frac{1+b t}{1+a t+b t+d t^{2}} \leq 1-\frac{(1-\delta) b}{d t} \leq e^{-(1-\delta) b /(d t)}
$$

and

$$
\beta=\frac{b t+d t^{2}}{1+a t+b t+d t^{2}}=1-\frac{1+a t}{1+a t+b t+d t^{2}} \leq 1-\frac{(1-\delta) a}{d t} \leq e^{-(1-\delta) a /(d t)} .
$$

Using these estimates along with (3.28) we see that for all $t$ sufficiently large, for all $1 \leq j \leq k$,

$$
\left|R_{j, k}(t, c)\right| \leq C e^{-k(1-\delta) a /(d t)} e^{-j(1-\delta) b /(d t)} \sum_{p=0}^{j} \frac{1}{(d t)^{2 p}}\left(\begin{array}{l}
j \\
p
\end{array}\right)\left(\begin{array}{l}
k \\
p
\end{array}\right)
$$

uniformly in $|c| \leq c_{3}$.

Suppose that $\sqrt{j k} /(d t)=n$. Then

$$
\begin{aligned}
& e^{-k(1-\delta) a /(d t)} e^{-j(1-\delta) b /(d t)} \\
& \quad=\exp (-(1-\delta)(a \sqrt{k / j}+b \sqrt{j / k}) n) \\
& \quad \leq \exp (-2(1-\delta) \sqrt{a b} n) \\
& \quad=\exp (-2(1-\delta) \sqrt{d+1} n)
\end{aligned}
$$

where, for the inequality we take the minimum of $a \theta+b / \theta$ and for the equality we use the fact that $a b=d+1$. Combined with (4.15) this shows that when $\sqrt{j k} /(d t)=n$

$$
\left|R_{j, k}(t, c)\right| \leq C j \exp (-2((1-\delta) \sqrt{d+1}-1) n) .
$$

Let $A_{n}=\{(j, k) \mid n \leq \sqrt{j k} /(d t)<n+1\}$. Then for any $M$

$$
\sum_{\sqrt{j k} /(d t) \geq M}\left|R_{j, k}(t, c)\right|=\sum_{n=M}^{\infty} \sum_{(j, k) \in A_{n}}\left|R_{j, k}(t, c)\right| .
$$

We note that the cardinality of $A_{n}$ is less than

$$
3 n d^{2} t^{2} \log ((n+1) d t) \text {. }
$$


This is because $(j, k) \in A_{n}$ implies that

$$
\frac{n^{2} d^{2} t^{2}}{j} \leq k \leq \frac{(n+1)^{2} d^{2} t^{2}}{j} \text { and } j \leq(n+1) d t
$$

and summing on $j$ we get (4.25).

It follows from (3.44) and (4.24) that

$$
\begin{aligned}
& \sum_{\sqrt{j k} /(d t) \geq M}\left|R_{j, k}(t, c)\right| \\
& \quad \leq C(d t)^{4} \sum_{n=M}^{\infty} n^{4} \exp (-2((1-\delta) \sqrt{d+1}-1) n) \\
& \quad \leq C \frac{(d t)^{4}}{(1-\delta) \sqrt{d+1}-1)} M^{4} \exp (-2((1-\delta) \sqrt{d+1}-1) M) .
\end{aligned}
$$

Clearly, there exists a constant $B$ such that when $M=B \log t$, this last term is $o(1)$ as $t \rightarrow \infty$.

\section{$5 \quad$ Proof of Theorem 1.4 when $\left(c_{1}, c_{2}\right)=(c, c)$ and $\mathrm{EG}_{1} \mathrm{G}_{2}>0$}

In this section we prove Theorem 1.4 in case 1 . and for $E G_{1} G_{2}>0$, by establishing the positivity conditions on the coefficients $R_{j, k}(t, c)$, (when $E G_{1} G_{2}=1$ ), as discussed in Remark 4.2. We pass to the case $E G_{1} G_{2}>0$ on page 41 ,

To proceed we need several estimates of parameters we are dealing with as $t \rightarrow \infty$. They follow from the definitions in (3.10)-(3.12).

Lemma 5.1 Ast $\rightarrow \infty$

$$
\begin{aligned}
1-\alpha & =\frac{b}{d t}-\frac{1+b^{2}}{(d t)^{2}}+O\left(t^{-3}\right) \\
1-\beta & =\frac{a}{d t}-\frac{1+a^{2}}{(d t)^{2}}+O\left(t^{-3}\right) \\
(1-\alpha)(1-\beta) & =\frac{d+1}{(d t)^{2}}-\frac{a\left(1+b^{2}\right)+b\left(1+a^{2}\right)}{(d t)^{3}}+O\left(t^{-4}\right) \\
\tilde{t}^{-2}=1-\theta & =\frac{1}{(d t)^{2}}-\frac{a+b}{(d t)^{3}}+O\left(t^{-4}\right)
\end{aligned}
$$




$$
\begin{aligned}
& \alpha^{j}=e^{-b j /(d t)+O\left(j^{2} / t^{2}\right)} \\
& \beta^{k}=e^{-a k /(d t)+O\left(k^{2} / t^{2}\right)}
\end{aligned}
$$

Also

$$
\begin{aligned}
-(d+2) \gamma+d(a+b) & =2((d+2)-(a+b))=-2 \zeta \\
a \gamma-d & =(a-1)^{2} \\
b \gamma-d & =(b-1)^{2} .
\end{aligned}
$$

\section{Proof}

$$
\begin{aligned}
1-\alpha & =\frac{1+b t}{1+a t+b t+d t^{2}} \\
& =\frac{1+b t}{d t^{2}} \frac{1}{1+a(d t)^{-1}+b(d t)^{-1}+d^{-1} t^{-2}} \\
& =\frac{1+b t}{d t^{2}}\left(1-a(d t)^{-1}-b(d t)^{-1}+O\left(t^{-2}\right)\right) \\
& =\frac{b}{d t}+\frac{d-b(a+b)}{d^{2} t^{2}}+O\left(t^{-3}\right) \\
& =\frac{b}{d t}-\frac{1+b^{2}}{d^{2} t^{2}}+O\left(t^{-3}\right)
\end{aligned}
$$

The rest of the lemma follows similarly.

Proof of Theorem 1.4 when $\mathbf{c}_{\mathbf{1}}=\mathbf{c}_{\mathbf{2}}=\mathbf{c}$. To begin let note that it is easy to see from Lemma 3.4, that $P_{j, k}(t) \geq 0$ for all $0 \leq j, k<\infty$, with the exception of $P_{0,0}(t)$. This must be the case because $\exp \left(P\left(a, b, \lambda_{1}, \lambda_{2}\right)\right)$ is the Laplace transform of an infinitely divisible random variable, as we remark following the proof of Lemma 2.1.

By (3.35)

$$
\begin{aligned}
Q_{j, 0}(t) & =-\frac{\left(\gamma t^{2}(\alpha-1)+t(2 \alpha-1)\right)}{\bar{t}^{2}} \alpha^{j-1} \\
& =-\frac{\left((-\gamma b+1) t+\gamma\left(1+b^{2}\right)-2 b+O(1 / t)\right)}{\bar{t}^{2}} \alpha^{j-1} \\
& =\frac{\left((b-1)^{2} t+2 b-\gamma\left(1+b^{2}\right)+O(1 / t)\right)}{\bar{t}^{2}} \alpha^{j-1} \\
& =\left(\frac{(b-1)^{2}}{d t}+O\left(1 / t^{2}\right)\right) \alpha^{j-1}
\end{aligned}
$$


Similarly

$$
Q_{0, k}(t)=\left(\frac{(a-1)^{2}}{d t}+O\left(1 / t^{2}\right)\right) \alpha^{k-1} .
$$

Thus we see that there exists a $t_{1}$ sufficiently large such that for all $t \geq t_{1}$, $R_{j, 0}(t, c)$ and $R_{0, k}(t, c)$ are both positive for all $j, k \geq 1$.

We now examine $R_{j, k}(t, c)$ for $j \wedge k \geq 1, j \leq k$. We write

$$
R_{j, k}(t, c)=\sum_{p=0}^{j} R_{j, k, p}(t, c) .
$$

Using (3.37) and (3.29) we see that

$$
\begin{aligned}
\frac{R_{j, k, p}(t, c)}{\alpha^{j-1} \beta^{k-1}} & \frac{\alpha \beta \widetilde{t}^{-2 p}}{\widetilde{t}^{2}}\left(\begin{array}{l}
j \\
p
\end{array}\right)\left(\begin{array}{l}
k \\
p
\end{array}\right) \frac{1}{p+1} \frac{(j-p)(k-p)}{j k}+\frac{c^{2} \widetilde{t}^{-2 p}}{\bar{t}^{2}}\left(\begin{array}{l}
j \\
p
\end{array}\right)\left(\begin{array}{l}
k \\
p
\end{array}\right) \\
& \left(-\gamma t^{2}\left((1-\alpha)(1-\beta)-\frac{(1-\beta) p}{j}-\frac{(1-\alpha) p}{k}+\widetilde{t}^{-2} \frac{j-p}{j} \frac{k-p}{k}\right)\right. \\
& \left.+t\left(\alpha\left(1-\beta-\frac{p}{k}\right)+\beta\left(1-\alpha-\frac{p}{j}\right)\right)\right) .
\end{aligned}
$$

When $p=0$ we get

$$
\begin{aligned}
& \frac{R_{j, k, 0}(t, c)}{\alpha^{j-1} \beta^{k-1}}=\frac{\alpha \beta}{\widetilde{t}^{2}}+\frac{c^{2}}{\bar{t}^{2}} \\
& \quad\left(-\gamma t^{2}\left((1-\alpha)(1-\beta)+\widetilde{t}^{-2}\right)+t(\alpha(1-\beta)+\beta(1-\alpha))\right)
\end{aligned}
$$

which is independent of $j, k$. Using Lemma 5.1 we see that

$$
\begin{aligned}
-\gamma t^{2} & \left((1-\alpha)(1-\beta)+\widetilde{t}^{-2}\right)+t(\alpha(1-\beta)+\beta(1-\alpha)) \\
& =-\frac{(d+2)}{d^{2}} \gamma+\frac{a+b}{d}+O\left(\frac{1}{t}\right) \\
& =\frac{-(d+2) \gamma+d(a+b)}{d^{2}}+O\left(\frac{1}{t}\right) \\
& =\frac{-2 \zeta}{d^{2}}+O\left(\frac{1}{t}\right) .
\end{aligned}
$$


Using this and Lemma 5.1 again we get

$$
\frac{R_{j, k, 0}(t, c)}{\alpha^{j-1} \beta^{k-1}}=\frac{1-2 c^{2}(\zeta / d)+O(1 / t)}{d^{2} t^{2}}
$$

where the $O(1 / t)$ term is independent of $j$ and $k$.

We now simplify the expression of the other coefficients $R_{j, k, p}(t, c), 1 \leq$ $p \leq j$. Set

$$
\frac{R_{j, k, p}(t, c)}{\alpha^{j-1} \beta^{k-1}}=\widetilde{t}^{-2 p}\left(\begin{array}{l}
j \\
p
\end{array}\right)\left(\begin{array}{l}
k \\
p
\end{array}\right)\left(\frac{1}{\widetilde{t}^{2}} F_{j, k, p}(t)+\frac{c^{2}}{\bar{t}^{2}} A_{j, k, p}(t)\right)
$$

where

$$
F_{j, k, p}(t)=\alpha \beta \frac{1}{p+1} \frac{(j-p)(k-p)}{j k}
$$

and

$$
\begin{aligned}
& A_{j, k, p}(t) \\
& =-\gamma t^{2}\left((1-\alpha)(1-\beta)-\frac{(1-\beta) p}{j}-\frac{(1-\alpha) p}{k}+\tilde{t}^{-2} \frac{j-p}{j} \frac{k-p}{k}\right) \\
& \quad+t\left(\alpha\left(1-\beta-\frac{p}{k}\right)+\beta\left(1-\alpha-\frac{p}{j}\right)\right) .
\end{aligned}
$$

Using Lemma 5.1 we have

$$
\begin{aligned}
& -\gamma t^{2}\left((1-\alpha)(1-\beta)-\frac{(1-\beta) p}{j}-\frac{(1-\alpha) p}{k}+\widetilde{t}^{-2} \frac{j-p}{j} \frac{k-p}{k}\right) \\
& =-\frac{\gamma}{d^{2}}\left(d+1-\frac{\left(b d t-\left(1+b^{2}\right)\right) p}{k}-\frac{\left(a d t-\left(1+a^{2}\right)\right) p}{j}+\frac{j-p}{j} \frac{k-p}{k}\right) \\
& =\frac{\gamma}{d^{2}}\left(-(d+2)+\frac{\left(b d t-b^{2}\right) p}{k}+\frac{\left(a d t-a^{2}\right) p}{j}-\frac{p^{2}}{j k}\right)+O\left(\frac{1}{t}\right) \\
& =\frac{\gamma}{d^{2}}\left(-(d+2)+\frac{b(d t-b) p}{k}+\frac{a(d t-a) p}{j}-\frac{p^{2}}{j k}\right)+O\left(\frac{1}{t}\right)
\end{aligned}
$$

and

$$
t\left(\alpha\left(1-\beta-\frac{p}{k}\right)+\beta\left(1-\alpha-\frac{p}{j}\right)\right)
$$




$$
\begin{aligned}
& =\frac{a}{d}\left(1+\frac{p}{j}\right)+\frac{b}{d}\left(1+\frac{p}{k}\right)-\frac{p t}{j}-\frac{p t}{k}+O\left(\frac{1}{t}\right) \\
& =\frac{1}{d}\left(a+b-\frac{p(d t-a)}{j}-\frac{p(d t-b)}{k}\right)+O\left(\frac{1}{t}\right) .
\end{aligned}
$$

In (5.14) and (5.15) the expressions $O(1 / t)$ are not necessarily the same from line to line. Nevertheless, it is important to note that they are independent of $p, j$ and $k$. That is there exists an $M>0$ such that all terms given as $O(1 / t)$ in (5.14) and (5.15) satisfy

$$
-\frac{M}{t}<O\left(\frac{1}{t}\right)<\frac{M}{t}
$$

This is easy to see since the $O(1 / t)$ terms, in addition to depending on $t$, depend on $a, b, p / j$ and $p / j \leq p / k \leq 1$.

Using (5.14), (5.15) we have

$$
\begin{aligned}
A_{j, k, p}(t)= & \frac{\gamma}{d^{2}}\left(-(d+2)+\frac{b(d t-b) p}{k}+\frac{a(d t-a) p}{j}-\frac{p^{2}}{j k}\right) \\
& +\frac{1}{d}\left(a+b-\frac{p(d t-a)}{j}-\frac{p(d t-b)}{k}\right)+O\left(\frac{1}{t}\right) \\
= & \frac{-(d+2) \gamma+d(a+b)}{d^{2}} \\
& +\frac{(\gamma a-d) p(d t-a)}{j d^{2}}+\frac{(\gamma b-d) p(d t-b)}{k d^{2}}-\frac{\gamma p^{2}}{j k d^{2}}+O\left(\frac{1}{t}\right) \\
= & \frac{-2 \zeta}{d^{2}}+\frac{(a-1)^{2} p(d t-a)}{j d^{2}}+\frac{(b-1)^{2} p(d t-b)}{k d^{2}}-\frac{\gamma p^{2}}{j k d^{2}}+O\left(\frac{1}{t}\right) .
\end{aligned}
$$

where, for the final equality we use (5.2).

Note that

$$
\begin{aligned}
B_{j, k, p}(t) & :=\frac{(a-1)^{2} p(d t-a)}{j}+\frac{(b-1)^{2} p(d t-b)}{k} \\
& \geq 2 p|(a-1)(b-1)| \frac{\sqrt{(d t-a)(d t-b)}}{\sqrt{j k}} \\
& =2 p|d+2-(a+b)| \frac{\sqrt{(d t-a)(d t-b)}}{\sqrt{j k}} .
\end{aligned}
$$


(For the inequality use $\alpha^{2}+\beta^{2} \geq 2 \alpha \beta$.) Therefore since $\zeta=a+b-(d+2)>0$,

$$
\begin{aligned}
A_{j, k, p}(t) & \geq \frac{2}{d^{2}}\left(p \frac{\sqrt{(d t-a)(d t-b)}}{\sqrt{j k}}-1\right) \zeta-\frac{\gamma p^{2}}{d^{2} j k}+O\left(\frac{1}{t}\right) \\
& =\frac{2}{d^{2}}\left(\frac{p(d t+O(1))}{\sqrt{j k}}-1\right) \zeta-\frac{\gamma p^{2}}{d^{2} j k}+O\left(\frac{1}{t}\right) \\
& =\frac{2}{d^{2}}\left(\frac{p d t}{\sqrt{j k}}\left(1+O\left(\frac{1}{t}\right)-\frac{\gamma p}{\zeta \sqrt{j k} d t}\right)-1\right) \zeta+O\left(\frac{1}{t}\right) .
\end{aligned}
$$

Thus we see that there exists a function $\epsilon_{t}$, depending only on $a$ and $b$ such that

$$
A_{j, k, p}(t) \geq \frac{2}{d^{2}}\left(\frac{p d t}{\sqrt{j k}}\left(1-\epsilon_{t}\right)-1\right) \zeta+O\left(\frac{1}{t}\right)
$$

where

$$
\lim _{t \rightarrow \infty} \epsilon_{t}=0,
$$

and, as we point out above the $O(1 / t)$ is independent of $p, j$ and $k$.

Remark 5.1 We interrupt this proof to make some comments which may be helpful in understanding what is going on. Note that if

$$
\frac{\sqrt{j k}}{d t} \leq 1-\widetilde{\epsilon} \quad \text { for some } \tilde{\epsilon}>0
$$

then

$$
R_{j, k}(t, c) \geq R_{j, k, 0}(t, c) \geq(1-\delta) \frac{1-2 c^{2}(\zeta / d)}{d^{2} t^{2}} \alpha^{j-1} \beta^{k-1} \quad \text { as } t \rightarrow \infty
$$

for all $\delta>0$. This follows from (5.10) and (5.20) since when (5.22) holds

$$
A_{j, k, p}(t) \geq \frac{2}{d^{2}}\left(\frac{1-\epsilon_{t}}{(1-\widetilde{\epsilon})}-1\right) \zeta+O\left(\frac{1}{t}\right)>0,
$$

for all $p \geq 1$, for all $t$ is sufficiently large. Consequently when (5.22) holds $R_{j, k}(t, c)>0$ for all $t$ is sufficiently large when

$$
c^{2}<\frac{d}{2 \zeta}
$$

(Here we also use (5.21) $)$ )

(When $\zeta \leq 0$, (5.23) shows that $R_{j, k}(t, c)>0$ for all $c \in R^{1}$. This is what we expect. (See the paragraph containing (2.12).) 
We use the next two lemmas to complete the proof of Theorem 1.4, in case 1 .

Lemma 5.2 For any $N_{0} \in R^{+}$, we can find $c_{0}>0$ and $t_{c_{0}}<\infty$ such that for all $t \geq t_{c_{0}}$

$$
R_{j, k, p}(t, c)>0
$$

for all $|c| \leq c_{0}$ and all $p, j$ and $k$ for which $j k / t \leq N_{0}$.

Proof This follows from (5.10) when $p=0$. Therefore, we can take $p \geq 1$.

We first show that for any $N \in R^{+}, R_{j, k, p}(t)>0$ when $\sqrt{j k}=N d t$, for all $t$ sufficiently large. By Remark 5.1 we can assume that $N \geq 1-\widetilde{\epsilon}$. It follows from (5.20) that

$$
A_{j, k, p}(t) \geq \frac{2}{d^{2}}\left(\frac{p}{N}\left(1-\epsilon_{t}\right)-1\right) \zeta+O\left(\frac{1}{t}\right),
$$

where $\epsilon_{t}$ satisfies (5.21). Therefore when $p \geq \Lambda N$ for any $\Lambda>1, A_{j, k, p}(t)>0$, and hence $R_{j, k, p}(t, c)>0$, for all $t$ sufficiently large.

Now suppose that

$$
p<\Lambda N \text {. }
$$

Since $\sqrt{j k}=N d t$ we see that

$$
\frac{\gamma p^{2}}{d^{2} j k} \leq \frac{\gamma \Lambda^{2}}{d^{4} t^{2}}=O\left(1 / t^{2}\right),
$$

where the $O(1 / t)$ term is independent of $p, j$ and $k$.

Note that by (5.1) and (5.12)

$$
\frac{1}{\widetilde{t}^{2}} F_{j, k, p}(t)=\frac{(j-p)(k-p)}{d^{2} t^{2}(p+1) j k}+O\left(\frac{1}{t^{3}}\right)
$$

Therefore, if in addition to (5.28) we also have $\Lambda N \leq j / 2$, so that $p<j / 2$, we see by (5.11), (5.17) and (5.29) that

$$
\begin{aligned}
\frac{1}{\tilde{t}^{2}} F_{j, k, p}(t)+\frac{c^{2}}{\bar{t}^{2}} A_{j, k, p}(t) \\
\quad \geq \frac{1}{d^{2} t^{2}}\left(\frac{(j-p)(k-p)}{(p+1) j k}-\frac{c^{2} 2 \zeta}{d}\right)+O\left(\frac{1}{t^{3}}\right) \\
\quad \geq \frac{1}{d^{2} t^{2}}\left(\frac{1}{4(p+1)}-\frac{c^{2} 2 \zeta}{d}\right)+O\left(\frac{1}{t^{3}}\right) \\
\quad \geq \frac{1}{d^{2} t^{2}}\left(\frac{1}{8 \Lambda N}-\frac{c^{2} 2 \zeta}{d}\right)+O\left(\frac{1}{t^{3}}\right) .
\end{aligned}
$$


Therefore we can obtain $R_{j, k, p}(t) \geq 0$ by taking

$$
c^{2} \leq \frac{d}{16 \Lambda^{\prime} N \zeta}
$$

for some $\Lambda^{\prime}>\Lambda$.

Now suppose that $\Lambda N>j / 2$. In this case we use (5.17) to see that

$$
A_{j, k, p}(t) \geq-\frac{2 \zeta}{d^{2}}+\frac{(a-1)^{2} p t}{2 d j}+O(1 / t) .
$$

It is easy to see that the right-hand side of (5.33) is greater than zero for all $t$ sufficiently large since

$$
\frac{(a-1)^{2} p t}{2 d j} \geq \frac{1}{4 d N \Lambda}(a-1)^{2} t .
$$

Thus we see that for any fixed $N, R_{j, k, p}(t)>0$ for all $t$ sufficiently large.

Since the $O(1 / t)$ terms are independent of $p, j$ and $k$ this analysis works for all $j$ and $k$ satisfying (5.26), and all $1 \leq p \leq j$ as long as (5.32) holds with $N$ replaced by $N_{0}$.

Lemma 5.3 For all $N_{0}$ and $B \in R^{+}$we can find a $c_{0}^{\prime}>0$ and $t_{c_{0}^{\prime}}<\infty$ such that for all $t \geq t_{c_{0}^{\prime}}$

$$
R_{j, k, p}(t, c)>0
$$

for all $|c| \leq c_{0}^{\prime}$ and all $0 \leq p \leq j \leq k$ for which

$$
N_{0} t \leq \sqrt{j k} \leq B t \log t .
$$

(The value of $N_{0}$ in Lemmas 5.2 and 5.3 can be taken as we wish. It will be assigned in the proof of this lemma.)

Proof By adjusting $N_{0}$ and $B$ we can replace (5.36) by the condition

$$
N_{0} \tilde{t} \leq \sqrt{j k} \leq B \tilde{t} \log \tilde{t} .
$$

Using (5.17), we see that if $j \leq \rho \widetilde{t}$

$$
A_{j, k, p}(t)=\frac{-2 \zeta}{d^{2}}+\frac{(a-1)^{2} p(d t-a)}{j d^{2}}+\frac{(b-1)^{2} p(d t-b)}{k d^{2}}
$$




$$
\begin{aligned}
& -\frac{\gamma p^{2}}{j k d^{2}}+O\left(\frac{1}{t}\right) \\
\geq & \frac{-2 \zeta}{d^{2}}+\frac{(a-1)^{2} p(d t-a)}{j d^{2}}-\frac{\gamma}{d^{2}}+O\left(\frac{1}{t}\right) \\
\geq & \frac{-2 \zeta}{d^{2}}+\frac{(a-1)^{2}}{2 \rho d}-\frac{\gamma}{d^{2}}+O\left(\frac{1}{t}\right) .
\end{aligned}
$$

Clearly, there exists a $\rho>0$, independent of $j$ and $k$ such that this term is positive. Thus we can assume that

$$
j \geq \rho \tilde{t}
$$

Furthermore, when $\sqrt{j k} / \widetilde{t}=N$, it follows from (3.28) that we can write (5.19) as

$$
A_{j, k, p}(t) \geq \frac{2}{d^{2}}\left(\frac{p}{N}\left(1+O\left(\frac{1}{t}\right)-\frac{\gamma}{\zeta(d t)^{2}}\right)-1\right) \zeta+O\left(\frac{1}{t}\right) .
$$

Let $\delta_{N}=(10 \log N / N)^{1 / 2}$. Clearly, if $p>\left(1+\delta_{N}\right) N$, the right-hand side of (5.40) is positive for all $t$ sufficiently large. Therefore, when $\sqrt{j k} / \widetilde{t}=N$, we may assume that

$$
p \leq\left(1+\delta_{N}\right) N
$$

(The value chosen for $\delta_{N}$ simplifies calculations made later in this proof.)

In addition we can also assume that

$$
p \geq p_{0}
$$

for any finite $p_{0}$, since if $p<p_{0}$

$$
F_{j, k, p}(t) \geq F_{j, k, p_{0}}(t) \geq c^{2} A_{j, k, p}(t) .
$$

for all $c>0$ sufficiently small.

We use the next lemma in the proof of Lemma 5.3.

Lemma 5.4 For $j \leq k$, with $p$ and $j$ large and $p / j$ small

$$
\begin{aligned}
\left(\begin{array}{l}
j \\
p
\end{array}\right) & \left(\begin{array}{l}
k \\
p
\end{array}\right)=\frac{1}{2 \pi p}\left(\frac{e^{2} j k}{p^{2}}\right)^{p} \\
& \exp \left(-\frac{p}{2 j}(p-1)-\frac{p}{2 k}(p-1)+O\left(p^{3} / j^{2}\right)\right)\left(1+O\left(p^{-1}\right)\right) .
\end{aligned}
$$


When $\tilde{t} \in R^{+}$is large and $\sqrt{j k} / \widetilde{t}=N$, under assumptions (5.39) and (5.41)

$$
\frac{1}{\widetilde{t}^{2 p}}\left(\begin{array}{l}
j \\
p
\end{array}\right)\left(\begin{array}{l}
k \\
p
\end{array}\right)=\frac{1}{2 \pi p}\left(\frac{e N}{p}\right)^{2 p}\left(1+O\left(p^{-1}\right)\right) .
$$

Proof By Stirlings's formula for integers $q$,

$$
q !=\sqrt{2 \pi} q^{q+1 / 2} e^{-q}\left(1+O\left(q^{-1}\right)\right)
$$

Therefore, since $j$ is large and $p / j$ is small, terms of the form

$$
\frac{\left(1+O\left(j^{-1}\right)\right)}{\left(1+O\left(p^{-1}\right)\right)\left(1+O\left((j-p)^{-1}\right)\right)}=\left(1+O\left(p^{-1}\right)\right) .
$$

Using this we see that

$$
\begin{aligned}
\left(\begin{array}{l}
j \\
p
\end{array}\right) & =\frac{j !}{(j-p) ! p !} \\
& =\frac{1}{\sqrt{2 \pi}} \frac{j^{j+1 / 2}}{(j-p)^{(j-p+1 / 2)} p^{p+1 / 2}}\left(1+O\left(p^{-1}\right)\right) \\
& =\frac{1}{\sqrt{2 \pi p}}\left(\frac{j}{p}\right)^{p} \frac{j^{j-p+1 / 2}}{(j-p)^{(j-p+1 / 2)}}\left(1+O\left(p^{-1}\right)\right) \\
& =\frac{1}{\sqrt{2 \pi p}}\left(\frac{j}{p}\right)^{p} \frac{1}{\left(1-\frac{p}{j}\right)^{(j-p+1 / 2)}}\left(1+O\left(p^{-1}\right)\right) \\
& =\frac{1}{\sqrt{2 \pi p}}\left(\frac{j}{p}\right)^{p} e^{-(j-p+1 / 2) \log (1-p / j)}\left(1+O\left(p^{-1}\right)\right) \\
& =\frac{1}{\sqrt{2 \pi p}}\left(\frac{j}{p}\right)^{p} e^{(j-p+1 / 2)\left(p / j+p^{2} /\left(2 j^{2}\right)+O\left(p^{3} / j^{3}\right)\right)}\left(1+O\left(p^{-1}\right)\right) \\
& =\frac{1}{\sqrt{2 \pi p}}\left(\frac{e j}{p}\right)^{p} e^{\left(-p^{2} /(2 j)+p /(2 j)+O\left(p^{3} / j^{2}\right)\right)}\left(1+O\left(p^{-1}\right)\right) \\
& =\frac{1}{\sqrt{2 \pi p}}\left(\frac{e j}{p}\right)^{p} e^{\left(-p(p-1) /(2 j)+O\left(p^{3} / j^{2}\right)\right)}\left(1+O\left(p^{-1}\right)\right) .
\end{aligned}
$$

Since this also holds with $j$ replaced by $k$ we get (5.44).

To get (5.45) we multiply each side of (5.44) by $\widetilde{t}^{-2 p}$ and substitute for $\sqrt{j k} / \widetilde{t}=d N$ and use the fact that under the assumptions (5.39) and (5.41),

$$
\frac{p^{3}}{j^{2}} \leq \frac{p^{2}}{j} \leq \frac{\left(1+\delta_{N}\right)^{2} N^{2}}{\rho t}
$$


Consequently, for all $t$ sufficiently large

$$
\exp \left(-\frac{p}{2 j}(p-1)-\frac{p}{2 k}(p-1)+O\left(p^{3} / j^{2}\right)\right)=1+O\left(N^{2} / t\right) .
$$

Proof of Lemma 5.3 continued We show that under the assumptions (5.39) and (5.41), when $\sqrt{j k} / \widetilde{t}=N$, for $N_{0} \leq N \leq B \log \widetilde{t}$, for any $0<B<$ $\infty$, and $t$ is sufficiently large,

$$
\frac{\widetilde{t}^{2}}{\alpha^{j-1} \beta^{k-1}} \sum_{p=p_{0}}^{\left(1+\delta_{N}\right) N} P_{j, k, p}(t) \geq C e^{2 N} \frac{1}{N^{3 / 2}}
$$

for some $C>0$, independent of $N$, and

$$
\frac{\bar{t}^{2}}{\alpha^{j-1} \beta^{k-1}} \sum_{p=p_{0}}^{\left(1+\delta_{N}\right) N} Q_{j, k, p}(t) \geq-D e^{2 N} \frac{1}{N^{3 / 2}}
$$

for some $D<\infty$, independent of $N$. If (5.51) and (5.52) hold, we can find a $c_{0}>0$ such that for all $c^{2} \leq c_{0}^{2}$

$$
\sum_{p=p_{0}}^{\left(1+\delta_{N}\right) N} R_{j, k, p}(t, c) \geq 0
$$

for all $t$ sufficiently large. Since we have already established that $A_{j, k, p}(t)>0$, when $p<p_{0}$ and $p>\left(1+\delta_{N}\right) N$, this completes the proof of Lemma 5.3.

Thus it only remains to prove (5.51) and (5.52). We do (5.51) first. It is considerably easier than (5.52). By Lemma 3.4 and (5.45)

$$
\begin{aligned}
& \frac{\widetilde{t}^{2}}{\alpha^{j-1} \beta^{k-1}} \sum_{p=p_{0}}^{\left(1+\delta_{N}\right) N} P_{j, k, p}(t) \\
& \quad=\sum_{p=p_{0}}^{\left(1+\delta_{N}\right) N} \frac{1}{\widetilde{t}^{2 p}}\left(\begin{array}{l}
j \\
p
\end{array}\right)\left(\begin{array}{l}
k \\
p
\end{array}\right) \frac{(j-p)(k-p)}{(p+1) j k} \\
& \geq C \sum_{p=p_{0}}^{\left(1+\delta_{N}\right) N} \frac{1}{\widetilde{t}^{2 p}}\left(\begin{array}{l}
j \\
p
\end{array}\right)\left(\begin{array}{l}
k \\
p
\end{array}\right) \frac{1}{p} \\
& \geq C \sum_{p=p_{0}}^{\left(1+\delta_{N}\right) N} \frac{1}{p^{2}}\left(\frac{e N}{p}\right)^{2 p} .
\end{aligned}
$$


In order to calculate this last sum we consider the function

$$
f_{m}(y)=\frac{1}{y^{m}}\left(\frac{e N}{y}\right)^{2 y}=\frac{1}{y^{m}} e^{2 y(1+\log N-\log y)}
$$

for $m \geq 0$ and $y \geq 2$. We have

$$
\begin{aligned}
f_{m}^{\prime}(y) & =\left(\frac{-m}{y}+2(1+\log N-\log y)-2\right) f(y) \\
& =\left(\frac{-m}{y}+2(\log N-\log y)\right) f(y) .
\end{aligned}
$$

This has a unique root $y_{m}$ where

$$
\log y_{m}+\frac{m}{2 y_{m}}=\log N .
$$

(Clearly, $\left.y_{0}=N\right)$. Let $y_{m}=N\left(1+\epsilon_{m}\right)$. Then

$$
\log \left(1+\epsilon_{m}\right)+\frac{m}{2 N\left(1+\epsilon_{m}\right)}=0 .
$$

Consequently

$$
\epsilon_{m}=-\frac{m}{2 N}+O\left(N^{-2}\right),
$$

which implies that

$$
y_{m}=N-\frac{m}{2}+O(1 / N) .
$$

Making use of the fact that $\left(-m / y_{m}+2\left(1+\log N-\log y_{m}\right)-2\right)=0$, we see that

$$
f_{m}^{\prime \prime}\left(y_{m}\right)=\left(\frac{m}{y_{m}^{2}}-\frac{2}{y_{m}}\right) f\left(y_{m}\right)<0 .
$$

Therefore

$$
\sup _{y \geq 2} f_{m}(y)=f_{m}\left(y_{m}\right) \leq \frac{1}{(N-m)^{m}} e^{2 N} .
$$

We also note that since $-m / y-2 \log y$ is increasing for $y>m / 2, f_{m}^{\prime}(y)$ is positive for $m / 2<y<y_{m}$ and negative for $y>y_{m}$. Consequently, $f_{m}(y)$ is unimodal. 
Now consider the last line of (5.54). The function being summed is $f_{2}(p)$. The above discussion shows that this function is unimodal, with a maximum at, at most, two points at which it is less than $2 e^{2 N} / N^{2}$. Consequently, to obtain (5.51) we can replace the sum in the last line of (5.54) by an integral and show that

$$
I_{1}:=\int_{p_{0}}^{\left(1+\delta_{N}\right) N} \frac{1}{r^{2}}\left(\frac{e N}{r}\right)^{2 r} d r \geq C e^{2 N} \frac{1}{N^{3 / 2}} .
$$

Making the change of variables $r=x N$ we have

$$
I_{1}=\frac{1}{N} \int_{p_{0} / N}^{1+\delta_{N}} \frac{1}{x^{2}}\left(\frac{e}{x}\right)^{2 x N} d x
$$

Recall that $N_{0} \leq N \leq 2 \log \tilde{t}$, and that we can take $N_{0}$ as large as we want, (but fixed and independent of $t$ ), and that $\delta_{N}=(10 \log N / N)^{1 / 2}$. Therefore

$$
\begin{aligned}
I_{1} & \geq \frac{1}{N} \int_{1-(10 \log N / N)^{1 / 2}}^{1+(10 \log N / N)^{1 / 2}} \frac{1}{x^{2}}\left(\frac{e}{x}\right)^{2 x N} d x \\
& \geq \frac{1}{2 N} \int_{1-(10 \log N / N)^{1 / 2}}^{1+(10 \log N / N)^{1 / 2}}\left(\frac{e}{x}\right)^{2 x N} d x .
\end{aligned}
$$

We write

$$
\left(\frac{e}{x}\right)^{2 x N}=\exp (2 x N(1-\log x))
$$

Set $x=1+y$ and note that for $|y|$ small

$$
\begin{aligned}
x(1-\log x) & =(1+y)(1-\log (1+y)) \\
& =(1+y)\left(1-\sum_{n=1}^{\infty}(-1)^{n-1} \frac{y^{n}}{n}\right) \\
& =1+y-\sum_{n=1}^{\infty}(-1)^{n-1} \frac{(1+y) y^{n}}{n} \\
& =1-\sum_{n=2}^{\infty}(-1)^{n-1} y^{n}\left(\frac{1}{n}-\frac{1}{n-1}\right) \\
& =1-\sum_{n=2}^{\infty}(-1)^{n} \frac{y^{n}}{n(n-1)} .
\end{aligned}
$$

When $|y| \leq(10 \log N / N)^{1 / 2}$, so that $|y|^{3} N<<1$, this shows that

$$
\begin{aligned}
\left(\frac{e}{x}\right)^{2 x N} & =e^{2 N} e^{-y^{2} N+O\left(y^{3} N\right)} \\
& =e^{2 N} e^{-y^{2} N}\left(1+O\left(|y|^{3} N\right)\right) .
\end{aligned}
$$


It follows from this that when we make the change of variables $x=1+y$ in (5.64) we get

$$
\begin{aligned}
I_{1} \geq & \frac{e^{2 N}}{2 N} \int_{-(10 \log N / N)^{1 / 2}}^{(10 \log N / N)^{1 / 2}} e^{-y^{2} N} d y \\
& \geq \frac{e^{2 N}}{2 \sqrt{2} N^{3 / 2}} \int_{-(20 \log N)^{1 / 2}}^{(20 \log N)^{1 / 2}} e^{-y^{2} / 2} d y .
\end{aligned}
$$

Since

$$
\int_{(20 \log N)^{1 / 2}}^{\infty} e^{-u^{2} / 2} d u \leq N^{-10}
$$

we see that (5.63) follows. Thus we have established (5.51).

Before proceeding to the proof of (5.52) we note that

$$
\sum_{p=p_{0}}^{\left(1+\delta_{N}\right) N} \frac{1}{\widetilde{t}^{2 p}}\left(\begin{array}{l}
j \\
p
\end{array}\right)\left(\begin{array}{l}
k \\
p
\end{array}\right) \leq \frac{e^{2 N}}{2 N^{1 / 2}} .
$$

To prove this we use (5.45) and the same argument that enables us to move from a sum to an integral that is given in (5.54)-(5.63), except that we use (5.62) with $m=1$. We continue and then use (5.67) to get

$$
\begin{aligned}
& \sum_{p=p_{0}}^{\left(1+\delta_{N}\right) N} \frac{1}{\widetilde{t}^{2 p}}\left(\begin{array}{l}
j \\
p
\end{array}\right)\left(\begin{array}{l}
k \\
p
\end{array}\right) \\
& \leq \int_{p_{0}}^{\left(1+\delta_{N}\right) N} \frac{1}{u}\left(\frac{e N}{u}\right)^{2 u} d u+O\left(\frac{e^{2 N}}{N}\right) \\
& \leq e^{2 N} \int_{0}^{\delta_{N}} e^{-y^{2} N} d x+O\left(\frac{e^{2 N}}{N}\right) \leq \frac{e^{2 N}}{2 N^{1 / 2}} .
\end{aligned}
$$

We now obtain (5.52). When $\sqrt{j k}=\widetilde{t} N$, by (5.11) and (5.40),

$$
\begin{aligned}
& \frac{\bar{t}^{2}}{\alpha^{j-1} \beta^{k-1}} Q_{j, k, p}(t) \\
& \quad=\frac{1}{\widetilde{t}^{2 p}}\left(\begin{array}{l}
j \\
p
\end{array}\right)\left(\begin{array}{l}
k \\
p
\end{array}\right) A_{j, k, p}(t) \\
& \quad \geq \frac{2}{d^{2} \widetilde{t}^{2 p}}\left(\begin{array}{l}
j \\
p
\end{array}\right)\left(\begin{array}{l}
k \\
p
\end{array}\right)\left\{2\left(\frac{p}{N}-1\right) \zeta+O\left(\frac{1}{t}\right)\right\} .
\end{aligned}
$$


By (5.71) we see that

$$
\sum_{p=p_{0}}^{\left(1+\delta_{N}\right) N} \frac{1}{\tilde{t}^{2 p}}\left(\begin{array}{l}
j \\
p
\end{array}\right)\left(\begin{array}{l}
k \\
p
\end{array}\right) O\left(\frac{1}{t}\right)=O\left(\frac{e^{2 N}}{t}\right)
$$

Therefore, to obtain (5.52), it suffices to show that for some $D<\infty$

$$
\sum_{p=p_{0}}^{\left(1+\delta_{N}\right) N} \frac{1}{\tilde{t}^{2 p}}\left(\begin{array}{l}
j \\
p
\end{array}\right)\left(\begin{array}{l}
k \\
p
\end{array}\right)\left(\frac{p}{N}-1\right) \geq-D e^{2 N} \frac{1}{N^{3 / 2}} .
$$

Here we use the fact that $N \leq B \log \widetilde{t}$ for some $0<B<\infty$.

Remark 5.2 Since the proof of (5.74) is rather delicate we make some heuristic comments to explain how we proceed. When $\sqrt{j k}=\widetilde{t} N$ the term $\tilde{t}^{-2 p}\left(\begin{array}{l}j \\ p\end{array}\right)\left(\begin{array}{l}k \\ p\end{array}\right)$, as a function of $p$, is $\exp (2 N)$ times values that are sort of normally distributed with mean $p=N$, and, roughly speaking,

$$
\sum_{p=p_{0}}^{\left(1+\delta_{N}\right) N} \frac{1}{\widetilde{t}^{2 p}}\left(\begin{array}{l}
j \\
p
\end{array}\right)\left(\begin{array}{l}
k \\
p
\end{array}\right) \sim C e^{2 N} \frac{1}{N^{1 / 2}}
$$

for all $t$ sufFiciently large. (In fact the upper bound is given in (5.71).) This is too large to enable us to get (5.74) so we must make use of the factors $\left(\frac{p}{N}-1\right)$, which is an odd function with respect to $p=N$, to get the cancellations that allow us to obtain (5.74). However, because we are canceling terms, we must take account of the error in Stirling's approximation; (see (5.46) ). To do this we need to show that the estimate in (5.74) remains the same even when we eliminate the terms in the summand that are not close to $N$.

Proof of Lemma 5.3 continued Note that by (5.45)

$$
\sum_{p=p_{0}}^{N\left(1-N^{-1 / 4}\right)} \frac{1}{{\widetilde{t^{2}}}^{2}}\left(\begin{array}{l}
j \\
p
\end{array}\right)\left(\begin{array}{l}
k \\
p
\end{array}\right) \leq C \sum_{p=p_{0}}^{N\left(1-N^{-1 / 4}\right)} f_{1}(p) .
$$

The fact that $f_{m}(y)$ is unimodal on $y>m / 2$ implies that $f_{1}(p)$ is increasing on the interval $\left[p_{0}, N\left(1-N^{-1 / 4}\right)\right]$. Therefore

$$
\sum_{p=p_{0}}^{N\left(1-N^{-1 / 4}\right)} \frac{1}{p}\left(\frac{e N}{p}\right)^{2 p} \leq C N f_{1}\left(N\left(1-N^{-1 / 4}\right)\right)
$$




$$
\begin{aligned}
& \leq C\left(\frac{e}{1-N^{-1 / 4}}\right)^{2 N\left(1-N^{-1 / 4}\right)} . \\
& =C e^{2 N\left(1-N^{-1 / 4}\right)\left(1-\log \left(1-N^{-1 / 4}\right)\right)} . \\
& \leq C e^{2 N\left(1-N^{-1 / 4}\right)\left(1+N^{-1 / 4}\right)}=C e^{2 N-2 N^{1 / 2}} .
\end{aligned}
$$

Let $\delta_{N}^{\prime}=N^{-1 / 4}$. The argument immediately above shows that to prove (5.74), it suffices to show that

$$
J_{1}:=\sum_{p=\left(1-\delta_{N}^{\prime}\right) N}^{\left(1+\delta_{N}\right) N} \frac{1}{\widetilde{t}^{2 p}}\left(\begin{array}{l}
j \\
p
\end{array}\right)\left(\begin{array}{l}
k \\
p
\end{array}\right)\left(\frac{p}{N}-1\right) \geq-D e^{2 N} \frac{1}{N^{3 / 2}} .
$$

By (5.45)

$$
J_{1}=\frac{1}{2 \pi} \sum_{p=\left(1-\delta_{N}^{\prime}\right) N}^{\left(1+\delta_{N}\right) N}\left(\frac{e N}{p}\right)^{2 p}\left(\frac{1}{N}-\frac{1}{p}\right)\left(1+O\left(\frac{1}{p}\right)\right) .
$$

Using (5.71) together with the fact that since $p \geq\left(1-\delta_{N}^{\prime}\right) N, 1 / p \leq 1 / N$, we see that

$$
\begin{aligned}
\sum_{p=\left(1-\delta_{N}^{\prime}\right) N}^{\left(1+\delta_{N}\right) N}\left(\frac{e N}{p}\right)^{2 p}\left(\frac{1}{N}-\frac{1}{p}\right) O\left(\frac{1}{p}\right) & \geq-\sum_{p=\left(1-\delta_{N}^{\prime}\right) N}^{\left(1+\delta_{N}\right) N}\left(\frac{e N}{p}\right)^{2 p} \frac{1}{p} O\left(\frac{1}{p}\right) \\
& \geq-C e^{2 N} \frac{1}{N^{3 / 2}} .
\end{aligned}
$$

Therefore, to obtain (5.78) that it suffices to show that

$$
\sum_{p=\left(1-\delta_{N}^{\prime}\right) N}^{\left(1+\delta_{N}\right) N}\left(\frac{e N}{p}\right)^{2 p}\left(\frac{1}{N}-\frac{1}{p}\right) \geq-D e^{2 N} \frac{1}{N^{3 / 2}} .
$$

In a minor modification of the analysis of $f_{m}(y)$, we write

$$
h(y):=\left(\frac{e N}{y}\right)^{2 y}\left(\frac{1}{N}-\frac{1}{y}\right)=\exp (2 y(1+\log N-\log y))\left(\frac{1}{N}-\frac{1}{y}\right) .
$$

Therefore

$$
h^{\prime}(y)=\left((2(1+\log N-\log y)-2)\left(\frac{1}{N}-\frac{1}{y}\right)+\frac{1}{y^{2}}\right)\left(\frac{e N}{y}\right)^{2 y} .
$$


Let $y=(1+\omega) N$. Then $h^{\prime}(y)=0$ when

$$
-2 \omega \log (1+\omega)+\frac{1}{N(1+\omega)}=0
$$

This equation is satisfied when

$$
\omega= \pm \frac{1}{\sqrt{2 N}}+O\left(\frac{1}{N}\right)
$$

Note that when $y=(1+\omega) N$

$$
\left(\frac{e N}{y}\right)^{y}=\left(\frac{e}{1+\omega}\right)^{(1+\omega) N} \leq e^{N}
$$

because $(e / x)^{x}$ is maximized when $x=1$. Therefore

$$
\left(\frac{e N}{y}\right)^{2 y}\left(\frac{1}{N}-\frac{1}{y}\right) \leq \frac{e^{2 N}}{N} \frac{\omega}{1+\omega}
$$

from which we get

$$
\sup _{1 \leq y \leq\left(1+\delta_{N}\right) N}|h(y)| \leq C\left(\frac{e^{2 N}}{N^{3 / 2}}\right) .
$$

It is easy to see that $h(y)$ is negative for $1 \leq y \leq N$ and that it decreases to its minimum value at $N(1-\omega)$ and then increases to zero at $y=N$. It then increases to its maximum value at $N(1+\omega)$ and then decreases for $N(1+\omega) \leq y \leq\left(1+\delta_{N}\right) N$. Consequently the difference between

$$
\sum_{p=\left(1-\delta_{N}^{\prime}\right) N}^{\left(1+\delta_{N}\right) N} h(p) \quad \text { and } \quad \int_{\left(1-\delta_{N}^{\prime}\right) N}^{\left(1+\delta_{N}\right) N} h(p) d p
$$

differs by at most $4 \max _{1 \leq p \leq\left(1+\delta_{N}\right) N}|h(p)|$. Since this is $O\left(e^{2 N} / N^{3 / 2}\right)$ by (5.87), and we are only trying to obtain (5.80), we can neglect this discrepancy. Therefore to obtain (5.80) we need only show that

$$
\int_{\left(1-\delta_{N}^{\prime}\right) N}^{\left(1+\delta_{N}\right) N} \frac{1}{p}\left(\frac{e N}{p}\right)^{2 p}\left(\frac{p}{N}-1\right) d p \geq-D^{\prime} \frac{e^{2 N}}{N^{3 / 2}} .
$$


Under the change of variables $p=x N$ the integral in (5.89) is equal to

$$
\int_{1-\delta_{N}^{\prime}}^{1+\delta_{N}} \frac{1}{x}\left(\frac{e}{x}\right)^{2 x N}(x-1) d x .
$$

By (5.66), in which $x=1+y$,

$$
\begin{aligned}
\left(\frac{e}{x}\right)^{2 x N} & =e^{2 N} e^{-y^{2} N+y^{3} N / 3+O\left(y^{4}\right) N} \\
& =e^{2 N} e^{-y^{2} N}\left(1+\frac{y^{3} N}{3}+O\left(y^{4}\right) N\right) .
\end{aligned}
$$

Therefore, with the change of variables $x=1+y$ we write the integral in (5.90) as

$$
e^{2 N} \int_{-\delta_{N}^{\prime}}^{\delta_{N}} \frac{y}{1+y} e^{-y^{2} N}\left(1+\frac{y^{3} N}{3}+O\left(y^{4}\right) N\right) d y .
$$

We use $(1+y)^{-1}=\left(1-y+y^{2}-y^{3}+O\left(y^{4}\right)\right)$ to write

$$
\begin{aligned}
& \frac{y}{1+y}\left(1+\frac{y^{3} N}{3}+O\left(y^{4}\right) N\right) \\
& \quad=y-y^{2}+y^{3}+\frac{y^{4} N}{3}-y^{4}+O\left(y^{5}\right) N .
\end{aligned}
$$

Using this we see that (5.92)

$$
=e^{2 N} \int_{-\delta_{N}^{\prime}}^{\delta_{N}} e^{-y^{2} N}\left(y-y^{2}+y^{3}+\frac{y^{4} N}{3}-y^{4}+O\left(y^{5}\right) N\right) d y .
$$

Recall that $\delta_{N}=(10 \log N / N)^{1 / 2}$ and $\delta_{N}^{\prime}=N^{-1 / 4}$. Since

$$
e^{2 N} \int_{(10 \log N / N)^{1 / 2}}^{\infty} e^{-y^{2} N} d y \leq \frac{e^{2 N}}{N^{10}}
$$

and

$$
e^{2 N} \int_{-\infty}^{-N^{-1 / 4}} e^{-y^{2} N} d y \leq e^{2 N-N^{1 / 2}},
$$

errors we can ignore in obtaining (5.52), we can simplify matters by replacing the integral in (5.94) by

$$
e^{2 N} \int_{-\infty}^{\infty} e^{-y^{2} N}\left(y-y^{2}+y^{3}+\frac{y^{4} N}{3}-y^{4}+O\left(y^{5}\right) N\right) d y
$$




$$
\begin{aligned}
& =-e^{2 N} \int_{-\infty}^{\infty} e^{-y^{2} N}\left(y^{2}-\frac{y^{4} N}{3}+y^{4}+O\left(y^{5}\right) N\right) d y \\
& =-e^{2 N} \int_{-\infty}^{\infty} e^{-y^{2}}\left(\frac{y^{2}}{N^{3 / 2}}-\frac{y^{4}}{3 N^{3 / 2}}+\frac{y^{4}}{N^{5 / 2}}+O\left(y^{5}\right) N^{-2}\right) d y \\
& =-\frac{e^{2 N}}{N^{3 / 2}} \int_{-\infty}^{\infty} e^{-y^{2}}\left(y^{2}-\frac{y^{4}}{3}\right) d y+O\left(\frac{e^{2 N}}{N^{2}}\right) .
\end{aligned}
$$

Since

$$
\begin{aligned}
& \frac{1}{\sqrt{\pi}} \int_{-\infty}^{\infty} e^{-y^{2}}\left(y^{2}-\frac{y^{4}}{3}\right) d y \\
& \quad=\frac{1}{\sqrt{2 \pi}} \int_{-\infty}^{\infty} e^{-y^{2} / 2}\left(\frac{y^{2}}{2}-\frac{y^{4}}{12}\right) d y \\
& \quad=\frac{1}{2}-\frac{1}{4}=\frac{1}{4},
\end{aligned}
$$

we obtain (5.89).

Proof of Theorem 1.4 when $c_{1}=c_{2}=c$ and $E_{1} G_{1}>0$ concluded Consider the Gaussian random variable $\left(G_{1} / \gamma, G_{2} / \gamma\right)$ where $\gamma=\left(E G_{1} G_{2}\right)^{1 / 2}$. This random variable has covariance $\Gamma$ in (2.1). By Lemma 5.3 there exists a $c_{0}^{\prime}>0$ such that $\left(G_{1} / \gamma+c, G_{2} / \gamma+c\right)$ has infinitely divisible squares for all $|c| \leq c_{0}^{\prime}$. Let $\widetilde{c}$ be the supremum of the $c_{0}^{\prime}$ for which this holds. Since, by hypothesis, (1.12) does not hold, $\widetilde{c}$ is finite. Therefore, $\left(G_{1} / \gamma+c, G_{2} / \gamma+c\right)$ has infinitely divisible squares for all $|c|<\widetilde{c}$ and not for any $c$ or which $|c|>\widetilde{c}$. Translating this into the notation used in Theorem 1.4 we have $\left(G_{1} / \gamma+c \alpha, G_{2} / \gamma+c \alpha\right)$ has infinitely divisible squares for all $|\alpha|<\widetilde{c} / c$ and not for any $|\alpha|$ for which $|\alpha|>\widetilde{c} / c$.

Therefore, to complete the proof of Theorem 1.4 when $c_{1}=c_{2}=c$ and $E G_{1} G_{2}>0$ we need only show that $\left(G_{1} / \gamma+c, G_{2} / \gamma+c\right)$ has infinitely divisible squares for $|c|=\widetilde{c}$. Consider the Laplace transform of $\left(G_{1} / \gamma+c, G_{2} / \gamma+c\right)$ in (3.1). Since it only depends on $c^{2}$ we can simplify the notation by taking $c>0$. Let $c_{m} \uparrow \widetilde{c}$. Abbreviate the third line of (3.1) by $\exp \left(P+c^{2} Q\right)$. Thus $\exp \left(P+c_{m}^{2} Q\right)$ is the Laplace transform of an infinitely divisible random variable. Therefore, for each $t>0$ the power series expansion of $P+c_{m}^{2} Q$ in $s_{1}$ and $s_{2}$ has positive coefficients, except for the constant term. Thus if we write

$$
P=\sum_{j, k} a_{j, k} s_{1}^{j} s_{2}^{k}, \quad Q=\sum_{j, k} b_{j, k} s_{1}^{j} s_{2}^{k}
$$


we see that $a_{j, k}+c_{m}^{2} b_{j, k} \geq 0$ for each $(j, k) \neq(0,0)$. Letting $c_{m} \uparrow \widetilde{c}$ we therefore have $a_{j, k}+\widetilde{c}^{2} b_{j, k} \geq 0$ for each $(j, k) \neq(0,0)$. This shows that $\exp \left(\left(P+\widetilde{c}^{2} Q\right)\right)$ is the Laplace transform of an infinitely divisible random variable.

Remark 5.3 In the remainder of this paper we continue to prove Theorem 1.4 for all $c_{1}, c_{2}$ and arbitrary covariance $E G_{1} G_{2}$. In each case, as immediately above, because (1.12) does not hold, there exists a $c^{\prime}<\infty$ such that $\left(G_{1}+\right.$ $\left.c c_{1}, G_{2}+c c_{2}\right)$ does not have infinitely divisible squares for all $c$ such that $|c|>c^{\prime}$. Therefore, if we can show that there exists some $c \neq 0$ for which both

$$
\left(G_{1}+c c_{1}, G_{2}+c c_{2}\right) \text { and }\left(G_{1}-c c_{1}, G_{2}-c c_{2}\right)
$$

have infinitely divisible squares, we can use the arguments in the preceding three paragraphs to show that there exists a critical point $\widetilde{c}$ such that $\left(G_{1}+\right.$ $\left.c c_{1}, G_{2}+c c_{2}\right)$ has infinitely divisible squares for all $|c| \leq \widetilde{c}$ and not for $|c|>\widetilde{c}$. Consequently, in the remainder of this paper, in which we consider different cases of $c_{1}, c_{2}$ and arbitrary covariance $E G_{1} G_{2}$ we will only show that (5.99) holds for some $c \neq 0$.

\section{Proof of Theorem 1.4 when $\left(c_{1}, c_{2}\right)=(c, \pm c)$}

We first assume that $E G_{1} G_{2}>0$ and that $\left(c_{1}, c_{2}\right)=(c,-c)$. In this case we have

$$
\begin{aligned}
E_{G} & \left(e^{-\left(\lambda_{1}\left(G_{1}+c\right)^{2}+\lambda_{2}\left(G_{2}-c\right)^{2}\right) / 2}\right) \\
& =\frac{1}{\left(H\left(a, b, \lambda_{1}, \lambda_{2}\right)\right)^{1 / 2}} \exp \left(-c^{2}\left(\frac{\rho \lambda_{1} \lambda_{2}+\lambda_{1}+\lambda_{2}}{2 H\left(a, b, \lambda_{1}, \lambda_{2}\right)}\right)\right),
\end{aligned}
$$

where $\rho=a+b+2$. This is exactly the same as (3.1) except that $\gamma$ is replaced by $\rho$. We now trace the proof in Sections 3 [5 and see what changes. Obviously much remains the same. In particular the power series $P$ is unchanged. The basic expression for $Q$ in (3.14) is essentially the same except that $\gamma$ is replaced by $\rho$. Thus Lemma 3.5 is also essentially the same except that $\gamma$ is replaced by $\rho$.

The analysis in Section 4 only uses the fact that $\gamma<\infty$, and since $\rho<\infty$, Lemma 4.1 also holds in this case. 
In going through Section 5 we see the coefficients of $Q$ change, but they still lead to essentially the same inequalities that allow us to complete the proof. In place of (5.2) we have

$$
\begin{aligned}
-3 \rho+a+b & =-2(3+(a+b)):=-2 \widetilde{\zeta} \\
a \rho-1 & =(a+1)^{2} \\
b \rho-1 & =(b+1)^{2} .
\end{aligned}
$$

Using this in (5.4) and (5.5), with $\gamma$ replaced by $\rho$, we get

$$
Q_{j, 0}(t)=\left(\frac{(b+1)^{2}}{d t}+O\left(1 / t^{2}\right)\right) \alpha^{j-1},
$$

and

$$
Q_{0, k}(t)=\left(\frac{(a+1)^{2}}{d t}+O\left(1 / t^{2}\right)\right) \alpha^{k-1} .
$$

We also see that we get (5.7) with $\gamma$ replaced by $\rho$ and consequently, in place of (5.10), we get

$$
\frac{R_{j, k, 0}(t, c)}{\alpha^{j-1} \beta^{k-1}}=\frac{1-2 c^{2}(\widetilde{\zeta} / d)+O\left(t^{-1}\right)}{d^{2} t^{2}} .
$$

Of course the key term in the proof is the analogue of $A_{j, k, p}(t)$. We get the third line of (5.17) with $\gamma$ replaced by $\rho$, which by (6.2) leads to (5.19) with $\zeta$ replaced by $\widetilde{\zeta}$ and $\gamma$ replaced by $\rho$. Therefore, all the subsequent lower bounds for $A_{j, k, p}(t)$ that are in Section 5 hold when $\zeta$ is replaced by $\widetilde{\zeta}$. In the proof of (5.52) in Section 5 the only property of $\zeta$ that is used is that is is positive. Since $\widetilde{\zeta}$ is also positive the same argument completes the proof of Lemma 5.3 and consequently, by Remark 5.3, of Theorem 1.4, when $E G_{1} G_{2}>0$ and $\left(c_{1}, c_{2}\right)=(c,-c)$.

When $E G_{1} G_{2}<0$ and $\left(c_{1}, c_{2}\right)=(c,-c)$ we note that

$$
\left(\left(G_{1}+c\right)^{2},\left(G_{2}-c\right)^{2}\right) \stackrel{\text { law }}{=}\left(\left(G_{1}+c\right)^{2},\left(-G_{2}+c\right)^{2}\right) .
$$

Now $E G_{1}\left(-G_{2}\right)>0$ and we are in the case proved on page 41. Therefore, by Remark 5.3, Theorem 1.4 holds in this case.

Finally when $E G_{1} G_{2}<0$ and $\left(c_{1}, c_{2}\right)=(c, c)$ we note that

$$
\left(\left(G_{1}+c\right)^{2},\left(G_{2}+c\right)^{2}\right) \stackrel{\text { law }}{=}\left(\left(G_{1}+c\right)^{2},\left(-G_{2}-c\right)^{2}\right) .
$$

Now $E G_{1}\left(-G_{2}\right)>0$ and we are in the case proved in the beginning of this section. 


\section{Proof of Theorem 1.4 when $\left(c_{1}, c_{2}\right)=(c, 0)$}

We first assume that $E G_{1} G_{2}>0$. In this case we have

$$
\begin{aligned}
E_{G} & \left(e^{-\left(\lambda_{1}\left(G_{1}+c\right)^{2}+\lambda_{2} G_{2}^{2}\right) / 2}\right) \\
& =\frac{1}{\left(H\left(a, b, \lambda_{1}, \lambda_{2}\right)\right)^{1 / 2}} \exp \left(-c^{2}\left(\frac{b \lambda_{1} \lambda_{2}+\lambda_{1}}{2 H\left(a, b, \lambda_{1}, \lambda_{2}\right)}\right)\right) .
\end{aligned}
$$

The term in the numerator of the exponential lacks the $\lambda_{2}$ that is present in (3.1) and (6.1). Therefore, the formulas for the coefficients of the power

series for the analogue of $Q$, which we denote by $\widetilde{Q}$, are different. It is easy to see that in place of (3.39) we get

$$
\begin{aligned}
& \widetilde{Q}\left(s_{1}, s_{2}, t\right)=-\frac{\left(b t^{2}\left(1-s_{1}\right)\left(1-s_{2}\right)+t\left(1-s_{1}\right)\right)}{\bar{t}^{2}} \\
&=-\sum_{n=0}^{\infty}\left(\alpha s_{1}+\beta s_{2}-\theta \alpha \beta s_{1} s_{2}\right)^{n}-\frac{\left(b t^{2}+t-\left(b t^{2}+t\right) s_{1}-b t^{2} s_{2}+b t^{2} s_{1} s_{2}\right)}{\bar{t}^{2}} \\
& \cdot \sum_{n=0}^{\infty}\left(\alpha s_{1}+\beta s_{2}-\theta \alpha \beta s_{1} s_{2}\right)^{n} .
\end{aligned}
$$

Using this, in place of Lemma 3.5, we get

Lemma 7.1 For all $t$ sufficiently large, and $j, k \geq 1$

$$
\widetilde{Q}_{j, 0}(t)=\frac{\left(b t^{2}+t\right)(1-\alpha)}{\bar{t}^{2}} \alpha^{j-1}
$$

and

$$
\widetilde{Q}_{0, k}(t)=\frac{b t^{2}(1-\beta)+\beta t}{\bar{t}^{2}} \beta^{j-1} .
$$

Furthermore, for all $t$ sufficiently large and for all $1 \leq j \leq k$

$$
\begin{aligned}
\widetilde{Q}_{j, k}(t) & \\
= & \frac{\alpha^{j-1} \beta^{k-1}}{\bar{t}^{2}} \sum_{p=0}^{j} \widetilde{t}^{-2 p}\left(\begin{array}{l}
j \\
p
\end{array}\right)\left(\begin{array}{l}
k \\
p
\end{array}\right) \\
& \left(-b t^{2}\left((1-\alpha)(1-\beta)-\frac{(1-\beta) p}{j}-\frac{(1-\alpha) p}{k}+\widetilde{t}^{-2} \frac{j-p}{j} \frac{k-p}{k}\right)\right. \\
& \left.+t \beta\left(1-\alpha-\frac{p}{j}\right)\right) .
\end{aligned}
$$


The analysis in Section 4 only uses the fact that $\gamma<\infty$. Since $b<\infty$, Lemma 4.1 also holds in this case.

In going through Section 5 we see the coefficients of $\widetilde{Q}$ change, but they still lead to similar inequalities that allow us to complete the proof. Using (7.3), (7.4) and (5.1) we get

$$
\widetilde{Q}_{j, 0}(t)=\left(\frac{b^{2}}{d^{2} t}+O(1 / t)\right) \alpha^{j-1}
$$

and

$$
\widetilde{Q}_{0, k}(t)=\left(\frac{d+1}{d^{2} t}+O(1 / t)\right) \beta^{k-1},
$$

since $a b=d+1$.

We next consider he analogue of (5.6) which we denote by $\widetilde{R}_{j, k}(t)$. We see that in computing this the first two lines of the analogue of (5.7) remain unchanged, except for replacing $c$ by 1 . The last two lines of (5.7) are now

$$
\begin{aligned}
& \left(-b t^{2}\left((1-\alpha)(1-\beta)-\frac{(1-\beta) p}{j}-\frac{(1-\alpha) p}{k}+\tilde{t}^{-2} \frac{j-p}{j} \frac{k-p}{k}\right)\right. \\
& \left.\quad+t \beta\left(1-\alpha-\frac{p}{j}\right)\right) .
\end{aligned}
$$

Therefore, in place of (5.10), we get

$$
\frac{\widetilde{R}_{j, k, 0}(t)}{\alpha^{j-1} \beta^{k-1}}=\frac{1-2 c^{2}(b / d)+O\left(t^{-1}\right)}{d^{2} t^{2}} .
$$

Using (5.14), with $\gamma$ replaced by $b$ and Lemma 5.1, we see that (7.8)

$$
\begin{aligned}
=- & \frac{b}{d^{2}}\left((a b+1)-\frac{b p(d t-b)}{k}-\frac{a p(d t-a)}{j}+\frac{p^{2}}{j k}\right) \\
& +\left(\frac{b}{d}-\frac{p(d t-a)}{d j}\right)+O\left(\frac{1}{t}\right) . \\
= & -\frac{2 b}{d^{2}}+\frac{p(d t-a)}{d^{2} j}+\frac{b^{2} p(d t-b)}{d^{2} k}-\frac{b p^{2}}{j k}+O\left(\frac{1}{t}\right) \\
\geq & \frac{2}{d^{2}}\left(p \frac{\sqrt{(d t-a)(d t-b)}}{\sqrt{j k}}-1\right) b-\frac{b p^{2}}{d^{2} j k}+O\left(\frac{1}{t}\right) .
\end{aligned}
$$


Comparing this inequality to the first line of (5.19) we see that we have exactly what we need to complete the proof in this case. The rest of the argument in Section 5 only uses the fact that $\zeta>0$. It is now replaced by $b>0$. Thus we get Lemma 5.3 and, by Remark 5.3, Theorem 1.4, when $\left(c_{1}, c_{2}\right)=(c, 0)$ and $E G_{1} G_{2}>0$. However this proof holds for $c$ positive or negative, so if $E G_{1} G_{2}<0$, we simply note that

$$
\left(G_{1}^{2},\left(G_{2}+c\right)^{2}\right) \stackrel{\text { law }}{=}\left(G_{1}^{2},\left(-G_{2}-c\right)^{2}\right) .
$$

Since $E G_{1}\left(-G_{2}\right)>0$ we are in the case just proved so, by Remark 5.3 , Theorem 1.4 holds in this case also.

\section{Proof of Theorem 1.4 for $\left(\mathbf{c}_{1}, \mathbf{c}_{2}\right) \in \mathbf{R}^{1} \times \mathbf{R}^{1}$}

It is simple to complete the proof from the results already obtained. Suppose neither $c_{1}$ nor $c_{2}$ are equal to zero. Then, clearly,

$$
\left(\left(G_{1}+c c_{1}\right)^{2},\left(G_{2}+c c_{2}\right)^{2}\right)
$$

is infinitely divisible, if and only if

$$
\left(\left(G_{1} / c_{1}+c\right)^{2},\left(G_{2} / c_{2}+c\right)^{2}\right)
$$

is infinitely divisible. We have already shown that there exists a critical point $\widetilde{c}>0$ such that

$$
\left(\left(G_{1} / c_{1}+c\right)^{2},\left(G_{2} / c_{2}+c\right)^{2}\right)
$$

is infinitely divisible for all $|c| \leq \widetilde{c}$ and not for $|c|>\widetilde{c}$. Consequently $\widetilde{c}$ is also a critical point for the infinite divisibility of

$$
\left(\left(G_{1}+c_{1} c\right)^{2},\left(G_{2}+c_{2} c\right)^{2}\right) .
$$

If $c_{1}=0$ we repeat this argument for

$$
\left(G_{1}^{2},\left(G_{2}+c c_{2}\right)^{2}\right)
$$




\section{References}

1. Bapat, R. (1989). Infinite divisibility of multivariate gamma distributions and $M$-matrices. Sankhya, 51, 73-78.

2. Eisenbaum, N. (2003). On the infinite divisibility of squared Gaussian processes. Prob. Theory Related Fields, 125, 381-392.

3. Eisenbaum, N. (2005). A connection between Gaussian processes and Markov processes. Elec. J. Probab., 10, 202-215.

4. Eisenbaum, N. and Kaspi, H. (2006). A characterization of the infinitely divisible squared Gaussian process. Ann. Probab., 34.

5. Eisenbaum, N., Kaspi, H., Marcus, M. B., Rosen, J., and Shi, Z. (2000). A Ray-Knight theorem for symmetric Markov processes. Ann. Probab., 28, 1781-1796.

6. W. Feller (1950). An Introduction to Probability Theory and its Applications, Vol. I, John Wiley, New York.

7. Griffiths, R. C. (1984). Characterizations of infinitely divisible multivariate gamma distributions. Jour. Multivar. Anal., 15, 12-20.

8. M. B. Marcus and J. Rosen (2006). Infinite divisibility of Gaussian Squares with non-zero means. submitted for publication.

9. M. B. Marcus and J. Rosen (2006). Markov Processes, Gaussian Processes and Local Times, Cambridge University Press, New York. 